\title{
Biological potential of thiazolidinedione derivatives of synthetic origin
}

\author{
Sucheta, Sumit Tahlan and Prabhakar Kumar Verma*
}

\begin{abstract}
Thiazolidinediones are sulfur containing pentacyclic compounds that are widely found throughout nature in various forms. Thiazolidinedione nucleus is present in numerous biological compounds, e.g., anti-malarial, antimicrobial, anti-mycobacterium, anticonvulsant, antiviral, anticancer, anti-inflammatory, antioxidant, anti-HIV (human immunodeficiency virus) and antitubercular agent. However, owing to the swift development of new molecules containing this nucleus, many research reports have been generated in a brief span of time. Therefore seems to be a requirement to collect recent information in order to understand the current status of the thiazolidinedione nucleus in medicinal chemistry research, focusing in particular on the numerous attempts to synthesize and investigate new structural prototypes with more effective antidiabetic, antimicrobial, antioxidant, anti-inflammatory, anticancer and antitubercular activity.
\end{abstract}

Keywords: Thiazolidinedione derivatives, Antidiabetic, Antimicrobial, Anti-inflammatory

\section{Introduction}

The number of antimicrobial drugs available in the market is vast, but there is a need to discover novel antimicrobial agents with better pharmacodynamic and pharmacokinetic properties with lesser or no side effects. Most of thiazolidinediones exhibit good bactericidal activity against various Gram-positive and Gram-negative bacteria. The bactericidal activity of thiazolidinediones derivatives depends on the substitution on the heterocyclic thiazolidine ring rather than the aromatic moiety.

Thiazolidinedione (Scheme 1) along with their derivatives draw attention as they have diverse biological as well as clinical use. Researchers focus on this moiety because it is involved in the control of various physiological activities. Heterocyclic moieties having Nitrogen and Sulfur are involved in a broad range of pharmacological processes. This created interest among researchers who have synthesized variety of thiazolidinediones derivatives and screened them for their various biological activities. In the present study, we have made an attempt to collect

${ }^{*}$ Correspondence: vermapk422@rediffmail.com

Department of Pharmaceutical Sciences, Faculty of Pharmaceutical

Sciences, Maharshi Dayanand University, Rohtak, Haryana 124001, India biological properties of thiazolidinediones and its derivatives of synthetic origin.

\section{Biological activities of thiazolidinediones derivatives in the new millennium}

Thiazolidinedione derivatives as antidiabetic agents

Diabetes mellitus (DM), also known as diabetes, is represented by the high blood sugar level over a period of prolonged time. There are three types of diabetes: (i) type $1 \mathrm{DM}$ in which pancreas fails to produce insulin. Previously, it was referred as "insulin-dependent diabetes mellitus" or "juvenile diabetes", (ii) type-2 DM a condition in which cells does not respond to insulin. Previously, it was referred as "non insulin-dependent diabetes mellitus", (iii) gestational diabetes is the third main type and arises in pregnant women with no prior record of diabetes with high blood sugar levels [1].

The fundamental reasons of diabetes are a low production of insulin, the inability of the body to use it, or a combination of both (hormone which regulate carbohydrate, fat and protein metabolism). Normally it is a long-standing syndrome having different clinical revelation, with a number of problems such as cardiovascular, hypertension, renal, neurological. It is a disease in which pancreas does not secrete sufficient insulin or cells 


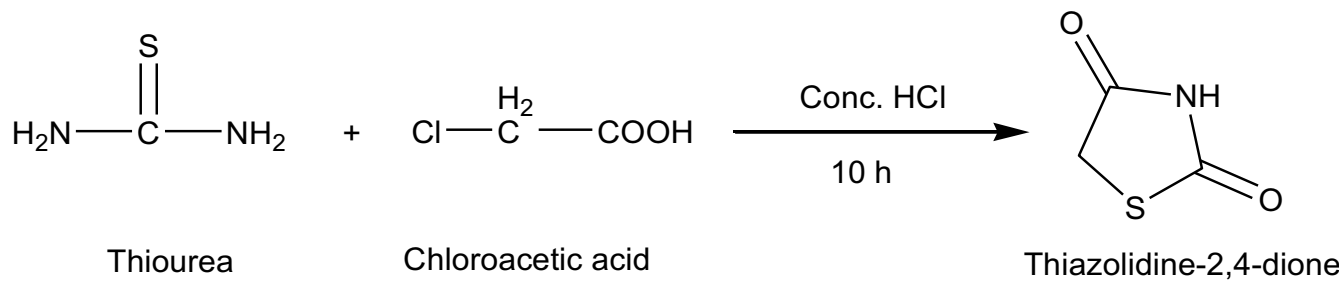

Scheme 1 Synthesis of Substituted thiazolidine-2,4-dione

prevent reacting toward secreted insulin, that's why cells cannot absorb blood glucose. Its symptoms are recurrent urination, tiredness, too much dehydration and hunger. It is cured by change in food habits, by regulation of proper diet; oral prescription and few situations include insulin injection $[2,3]$. The thiazole moiety is a significant heterocyclic unit in drug invention. Literature survey shows that the wide-spread studies have been carried out on the production of thiazolidinediones. Thiazolidiones compounds shows a number of pharmacological activities such as antimicrobial, antitubercular, anti-tumor, anti-viral, anti-HIV, anti-inflammatory and anti-diabetic effects [4-6].

Datar et al. [7] synthesized a new series of thiazolidinediones by the reaction of thiazolidenedione with several benzaldehyde derivatives using Scheme 2. In vitro anti-diabetic activity of synthesized compound was performed by SLM model. In this series compounds $\mathbf{1}$ and 2 found to be most active [5-(3,4-dimethoxy)benzylidine2,4-thiazolidinedione,5-(3,4,5 trimethoxy)benzylidine2,4-thiazolidenedione] due to presence of methoxy group and comparable to standard drug pioglitazone studies. The results of the most active compound are indicated Tables 1 and 2 (Datar et al. [7]).

Swapna et al. [8] synthesized novel thiazolidinediones by using Scheme 3. In vitro antidiabetic activity performed by alloxan induced tail tipping method. From this series compound $\mathbf{3}, \mathbf{4}, \mathbf{5}$ showed highest activity as comparable to standard drug metformin because of presence of electron donating group. The results of most active derivatives showed in Table 3 (Swapna et al. [8]).

Pattan et al. [2] synthesized a new series of thiazolidinediones derivatives [5-(4-substitutedsulfonylbenzylidene)2,4-thiazolidinedione] using Scheme 4. The In vitro antidiabetic activity performed by ANOVA and Dunnet's ' $\mathrm{t}$ ' test. From this series $\mathbf{6 , 7}$ and $\mathbf{8}$ compound showed moderates activity and comparable to the standard drug glibenclamide. The results of active compound are given in Table 4 (Pattan et al. [2]).

Badiger et al. [9] synthesized novel thiazolidinediones derived from 4-fluorophenylacetic acid and thiosemicarbazide in phosphorous oxychloride using Scheme 5. The

Chloro acetic
acid


Table 1 Blood glucose level in experimental animals (mg/dl)

\begin{tabular}{llllll}
\hline Compounds & \multicolumn{5}{l}{ Time (min) } \\
\cline { 2 - 6 } & $\mathbf{0}$ & $\mathbf{3 0}$ & $\mathbf{6 0}$ & $\mathbf{9 0}$ & $\mathbf{1 2 0}$ \\
\hline DMSO & 145 & 150 & 150 & 147 & 141 \\
Pioglitazone & 139 & 105 & 110 & 112 & 115 \\
$\mathbf{1}$ & 141 & 112 & 117 & 118 & 112 \\
$\mathbf{2}$ & 147 & 110 & 112 & 107 & 104 \\
\hline
\end{tabular}

Table 2 Decrease in blood glucose levels by AUC method

\begin{tabular}{llllll}
\hline Compounds & \multicolumn{2}{l}{ Time $(\mathbf{m i n})$} \\
\cline { 2 - 6 } & $\mathbf{3 0}$ & $\mathbf{6 0}$ & $\mathbf{9 0}$ & $\mathbf{1 2 0}$ & $\begin{array}{l}\text { \% reduction in blood } \\
\text { glucose level }\end{array}$ \\
\hline DMSO & +11 & +05 & +02 & -04 & +31 \\
Pioglitazone & -34 & -39 & -29 & -26 & -23.07 \\
$\mathbf{1}$ & -29 & -25 & -24 & -27 & -21.71 \\
$\mathbf{2}$ & -37 & -35 & -28 & -24 & -22.84 \\
\hline
\end{tabular}

Table 3 Blood glucose level $(\mathrm{mg} / \mathrm{dl})$ of synthesized thiazolidinediones derivatives

\begin{tabular}{lrrr}
\hline Compounds & \multicolumn{3}{c}{ Blood glucose level (mean $\mathbf{\text { SE}}$ ) } \\
\cline { 2 - 4 } & \multicolumn{1}{c}{$\mathbf{h}$} & $\mathbf{3} \mathbf{h}$ & \multicolumn{1}{c}{$\mathbf{~ h}$} \\
\hline $\mathbf{3}$ & $343 \pm 5.797$ & $313.8 \pm 9.411$ & $303.2 \pm 9.827$ \\
$\mathbf{4}$ & $341.5 \pm 6.158$ & $320.5 \pm 6.737$ & $313 \pm 9.500$ \\
$\mathbf{5}$ & $353.7 \pm 6.026$ & $315.8 \pm 8.109$ & $311.2 \pm 9.297$ \\
Positive control & $335.7 \pm 5.168$ & $345.5 \pm 5.488$ & $354 \pm 8.135$ \\
Normal control & $125.0 \pm 4.497$ & $126.3 \pm 4.047$ & $127.7 \pm 3.703$ \\
Metformin & $343.3 \pm 6.206$ & $322.8 \pm 4.989$ & $292.0 \pm 7.767$ \\
\hline
\end{tabular}

in vitro antidiabetic activity of synthesized compound [5-\{[2-(4-alkyl/aryl)-6-arylimidazo[1,2][1,3,4] thiadiazol5-yl]metylene\}-1,3-thiazolidine-2,4-dione] were performed by alloxan induced tail tipping method. Among them, compounds $\mathbf{9}$ and $\mathbf{1 0}$ found to be most active due to presence of napthyl and coumarinyl groups at $\mathrm{C}_{5}$

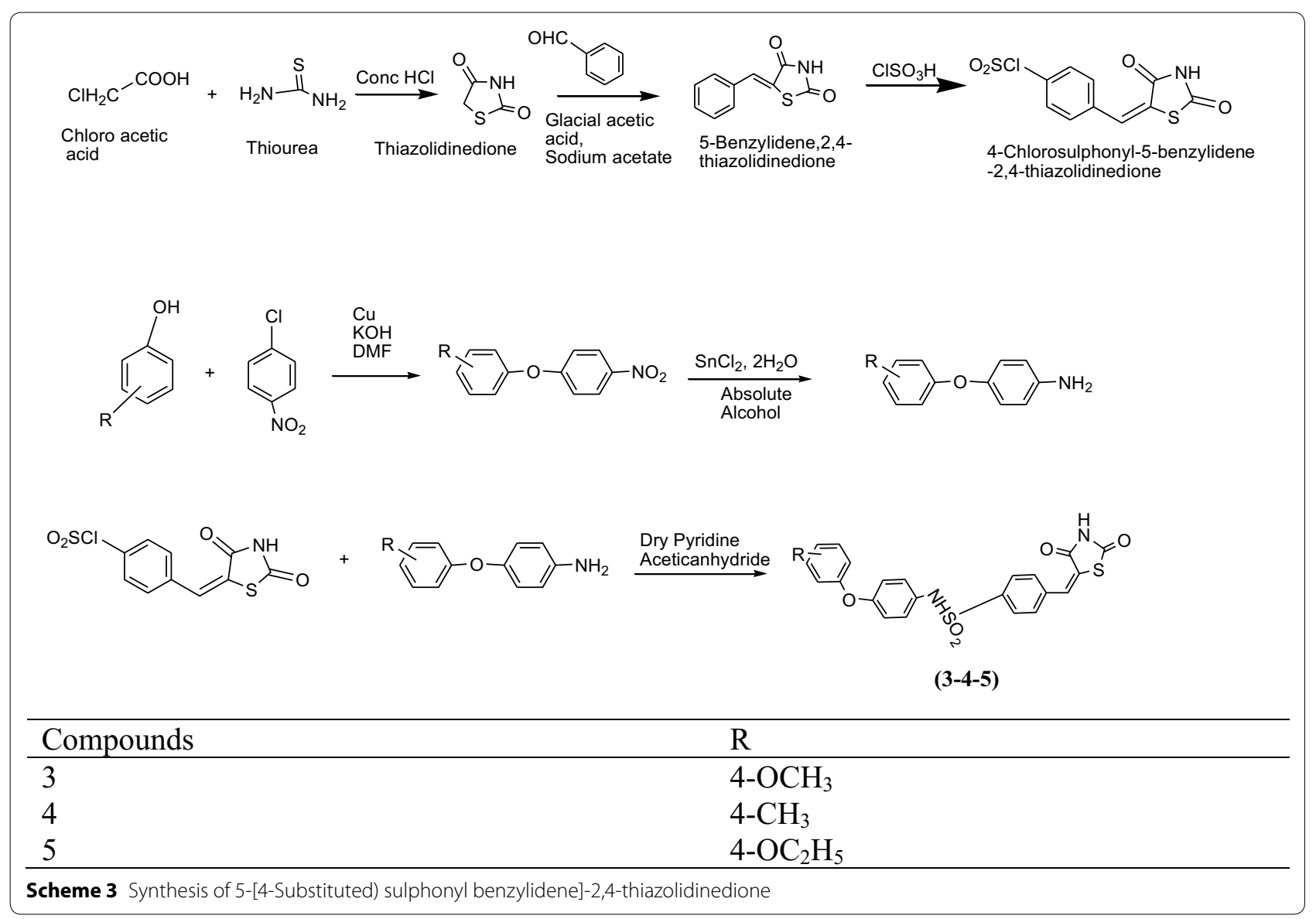




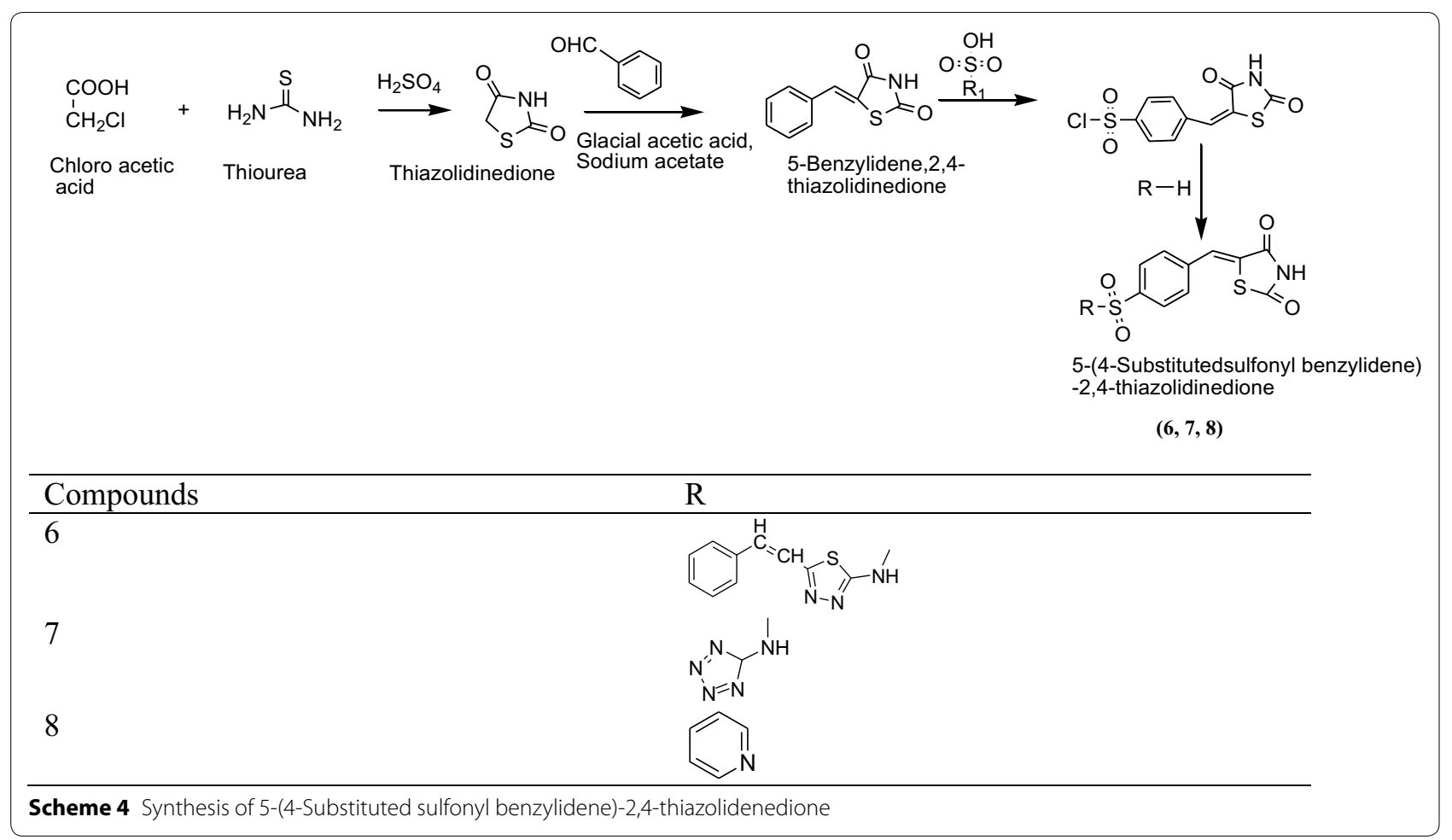

Table 4 Blood glucose level ( $\mathrm{mg} / \mathrm{dl}$ ) in synthesized compounds

\begin{tabular}{|c|c|c|c|c|}
\hline \multirow[t]{2}{*}{ Compounds } & \multicolumn{4}{|c|}{ Blood glucose level (mean \pm SE) } \\
\hline & $\mathrm{Oh}$ & $1 \mathrm{~h}$ & $3 \mathrm{~h}$ & $6 \mathrm{~h}$ \\
\hline 6 & $320.5 \pm 15.81$ & $145.5 \pm 2.26$ & $137.0 \pm 3.80$ & $123.5 \pm 1.10$ \\
\hline 7 & $213.5 \pm 8.78$ & $140.7 \pm 3.30$ & $106.3 \pm 6.91$ & $95.75 \pm 6.06$ \\
\hline 8 & $283.5 \pm 43.76$ & $205.75 \pm 49.7$ & $166.3 \pm 38.92$ & $124.5 \pm 13.16$ \\
\hline Standard & $385.8 \pm 21.37$ & $230.8 \pm 12.35$ & $156.8 \pm 10.87$ & $93.4 \pm 4.98$ \\
\hline
\end{tabular}

position as compared to standard drug pioglitazone. The results of synthesized compounds presented in Table 5 (Badiger et al. [9]).

Patil et al. [10] synthesized a new series of thiazolidinedione derivatives derived from thiourea and chloroacetic acid in ethanol/DMF as presented in Scheme 6. The In vitro antidiabetic activity of synthesized compounds was performed by alloxan induced tail tipping method. From these series compounds 11, 12 and 13 showed better activities compared to pioglitazone and metformin as standard drug. The results of most active derivatives showed in Table 6 (Patil et al. [10]).

Srikanth et al. [11] synthesized an innovative sequence of thiazolidinediones using 4-fluoroaniline, methyl acrylate and thiourea using proper solvent as showed in Scheme 7. The In vitro antidiabetic activities of synthesized compounds were confirmed by tail vein method and ANOVA method. In this series compounds 14, 15, 16 and 17 showed significant activity as compared to standard drug rosiglitazone. The results of synthesized compounds presented in Table 7 (Srikanth et al. [11]). 


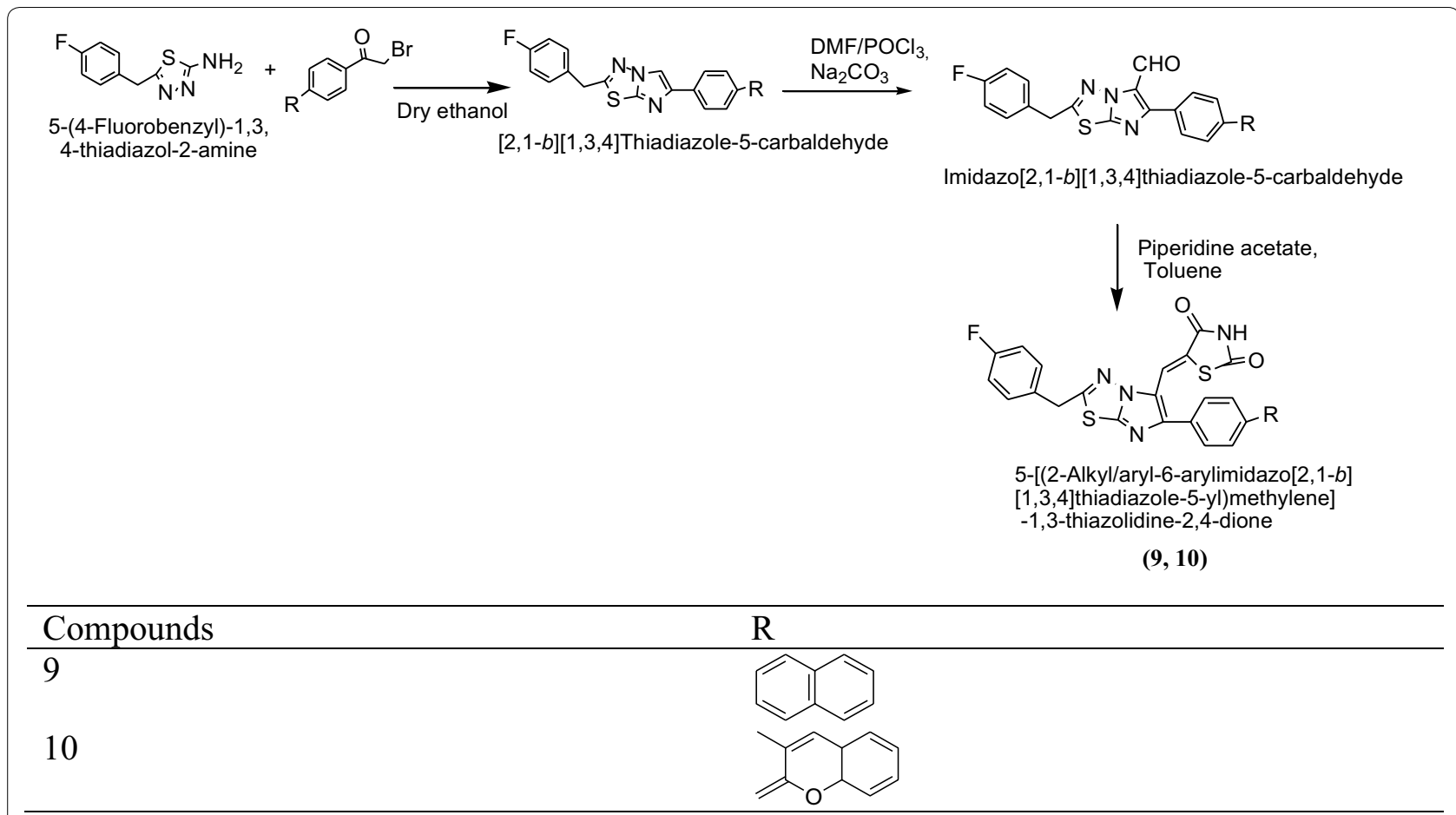

Scheme 5 Synthesis of 5-\{[2-(4-Fluorobenzyl)-6-arylimidazo[2,1-b] [1, 3, 4] thiadiazol-5-yl]methylene\}thiazolidine-2,4-diones

Table 5 Plasma glucose level of 3-4 at various drug doses

\begin{tabular}{llll}
\hline Compounds & \multicolumn{3}{l}{$\begin{array}{l}\text { \% decrease in plasma glucose level (PG) at vari- } \\
\text { ous drug doses (mg/kg bodyweight) }\end{array}$} \\
\cline { 2 - 4 } & $\mathbf{1 0} \mathbf{~ m g}$ & $\mathbf{3 0} \mathbf{~ m g}$ & $\mathbf{6 0} \mathbf{~ m g}$ \\
\hline $\mathbf{9}$ & $42.48+3.25$ & $62.24+3.42$ & $70.35+3.14$ \\
$\mathbf{1 0}$ & $45.42+1.25$ & $58.36+2.36$ & $68.42+2.16$ \\
Pioglitazone & $47.25+5.50$ & $64.59+5.42$ & $75.43+3.40$ \\
\hline
\end{tabular}

Nikalje et al. [12] designed few thiazolidinediones derivatives from thiazolidindione via 4-hydroxy, 3-ethoxy benzaldehyde in ethanol, benzoic acid and piperidine using Scheme 8. The In vitro antidiabetic activity of synthesized compounds was confirmed by ANOVA, alloxan induced diabetic rat model and dunnet' $t$ test. From this series compounds 18, 19, 20, 21, and 22 showed better activity as compared to standard drug rosiglitazone. The results of synthesized compounds presented in Table 8 (Nikalje et al. [12]).

Jiwane et al. [13] synthesized a new series of thiazolidine-2,4-dione derivatives from 5-(benzylidene)thiazolidine-2,4-dione with $N N^{1}$-dimethylformamide in diethyl amino as presented in Scheme 9. The In vitro anitdiabetic activity of synthesized compound [3-((diethyl amino) methyl)-5-(4-methoxybenzylidine)thiazolidine-2,4-dione] were confirmed by alloxan induced diabetic rat model. From this series, compounds 23 and $\mathbf{2 4}$ showed remarkable activity as that of the standard rosiglitazine, which indicates that the substitution of $\alpha$-amino methyl group at position-3 show different hypoglycemic activity. The results of most active derivatives showed in Table 9 (Jiwane et al. [13]).

Grag et al. [14] designed novel thiazolidinediones derivative from 3-benzylthiazolidine-2,4-dione with selected various substituted aromatic aldehydes in ethanol, benzoic acid and piperidine using Scheme 10. In vitro antidiabetic activity of synthesized compound 


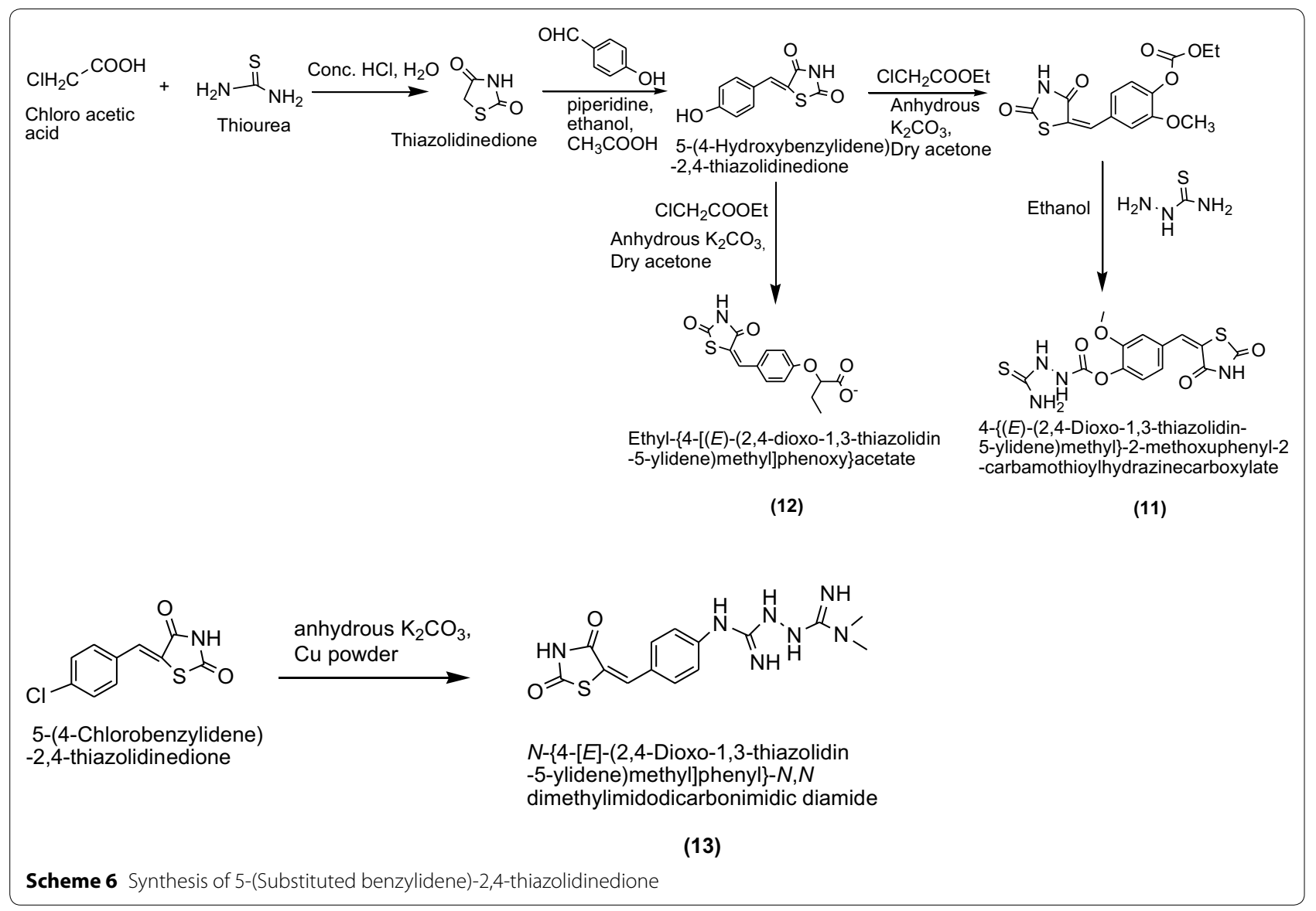

Table 6 Hypoglycemic effect of synthesized compounds

\begin{tabular}{|c|c|c|c|c|}
\hline \multirow[t]{2}{*}{ Compounds } & \multicolumn{4}{|c|}{ Blood glucose level mg/dl (mean $\pm \mathrm{SE}$ ) } \\
\hline & $\mathrm{Oh}$ & $3 \mathrm{~h}$ & $6 \mathrm{~h}$ & $24 \mathrm{~h}$ \\
\hline 11 & $376.4 \pm 21.00$ & $342.8 \pm 21.58$ & $315.2 \pm 21.66$ & $276 \pm 21.79$ \\
\hline 12 & $326.2 \pm 25.32$ & $300 \pm 25.03$ & $278.2 \pm 25.76$ & $245.2 \pm 25.91$ \\
\hline 13 & $355 \pm 24.59$ & $322.8 \pm 24.10$ & $253.8 \pm 23.45$ & $231.4 \pm 23.48$ \\
\hline Pioglitazone & $402.2 \pm 28.7$ & $363.4 \pm 26.08$ & $302.4 \pm 26.87$ & $232.2 \pm 20.53$ \\
\hline Metformin & $441.8 \pm 18.71$ & $399.4 \pm 17.72$ & $289.4 \pm 18.46$ & $219.6 \pm 18.40$ \\
\hline Vehicle control & $304.2 \pm 36.81$ & $308.2 \pm 36.85$ & $309 \pm 37.92$ & $310.4 \pm 39.57$ \\
\hline Diabetic control & $322.2 \pm 22.96$ & $337 \pm 23.59$ & $347 \pm 24.01$ & $363.4 \pm 24.0$ \\
\hline Normal control & $120.33 \pm 7.76$ & $125.66 \pm 2.08$ & $126.66 \pm 3.05$ & $129.33 \pm 1.52$ \\
\hline
\end{tabular}




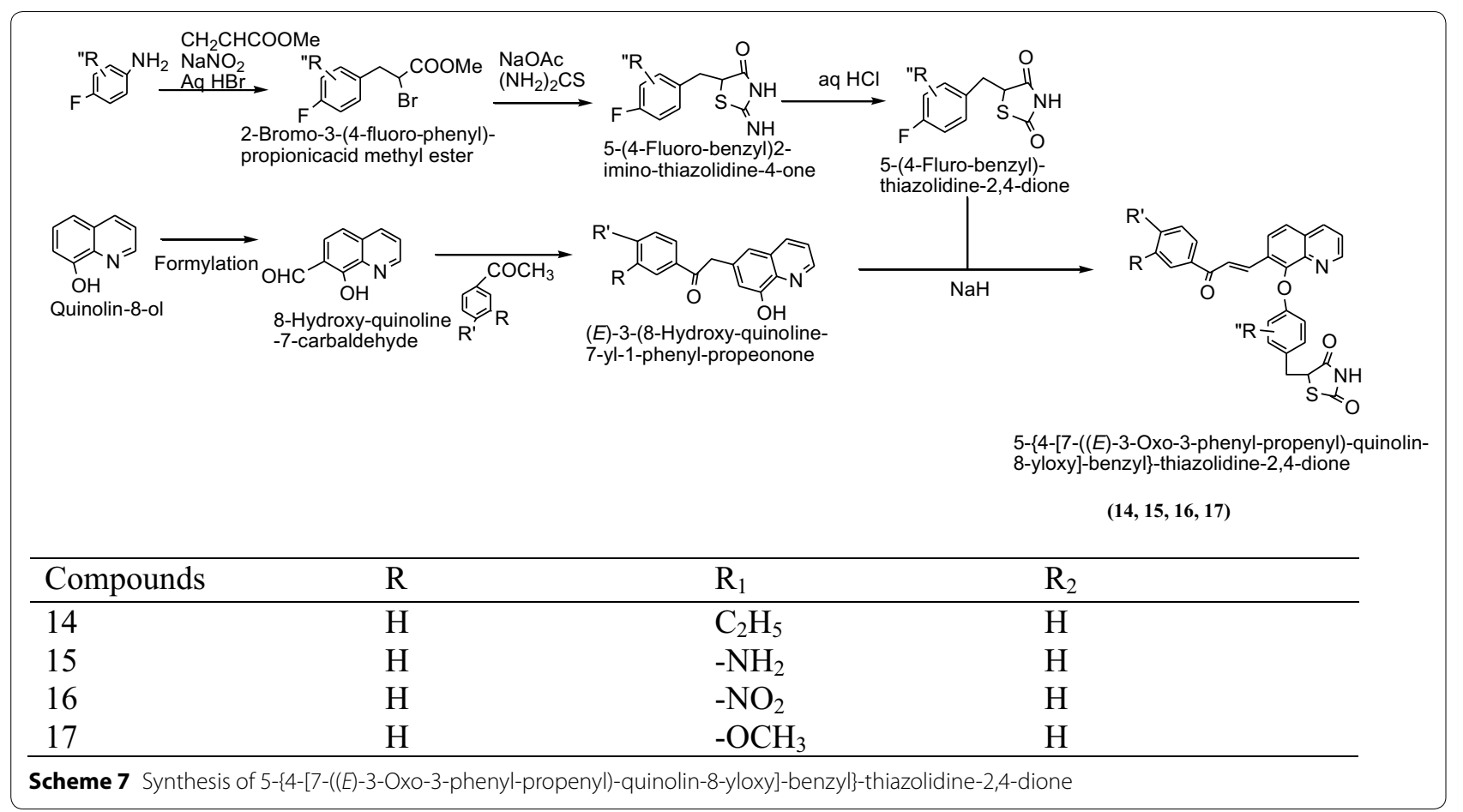

Table 7 Antidiabetic activities of synthesized compounds (mg/dl)

\begin{tabular}{ll}
\hline Compounds & Blood glucose level (mean $\pm \mathbf{S E})$ \\
\hline $\mathbf{1 4}$ & $82.81 \pm 1.115$ \\
$\mathbf{1 5}$ & $86.31 \pm 0.993$ \\
$\mathbf{1 6}$ & $87.21 \pm 1.233$ \\
$\mathbf{1 7}$ & $97.91 \pm 1.870$ \\
Rosiglitazone & $65.58 \pm 1.013$ \\
\hline
\end{tabular}

[5-arylidene-3-benzyl-thiazolidine-2,4-diones] was confirmed by ANOVA, alloxan induced diabetic rat model and dunnet' $t$ test. From this series compounds 25, 26 and 27 showed highest activity because of methoxy group as compared to standard rosiglitazone. The results of synthesized compounds presented in Table 10 (Grag et al. [14]).
Bhat et al. [15] synthesized a new series of thiazolidinediones derivatives derived from 5-arylidene2,4-thiazolidinedione using Scheme 11. The In vitro antidiabetic activity of synthesized compound [5-(4-methoxy-benzylidene)-2,4-dioxo-thiazolidin-3-yl]acetic acid] and [5-(substituted)-2,4-dioxo-thiazolidin3-yl]-acetic acid substituted ester were performed by alloxan induced tail tipping method and SLM. Among them compounds $28,29,30,31,32,33,34,35$ and 36 found to be most active or higher than rosiglitazone and metformin using as standard drug. The results of most active derivatives showed in Table 11 (Bhat et al. [15]).

Jawale et al. [16] synthesized innovative chain of thiazolidinediones derived from maleic anhydride and thiourea was treated with water using Scheme 12. The In vitro antidiabetic activity of synthesized compounds was performed by alloxan induced tail tipping method using wister rat, dunnet' $t$ test and SLM model. Among them compounds $37,38,39$ and $\mathbf{4 0}$ found to be significant activity metformin using as standard drug. The 


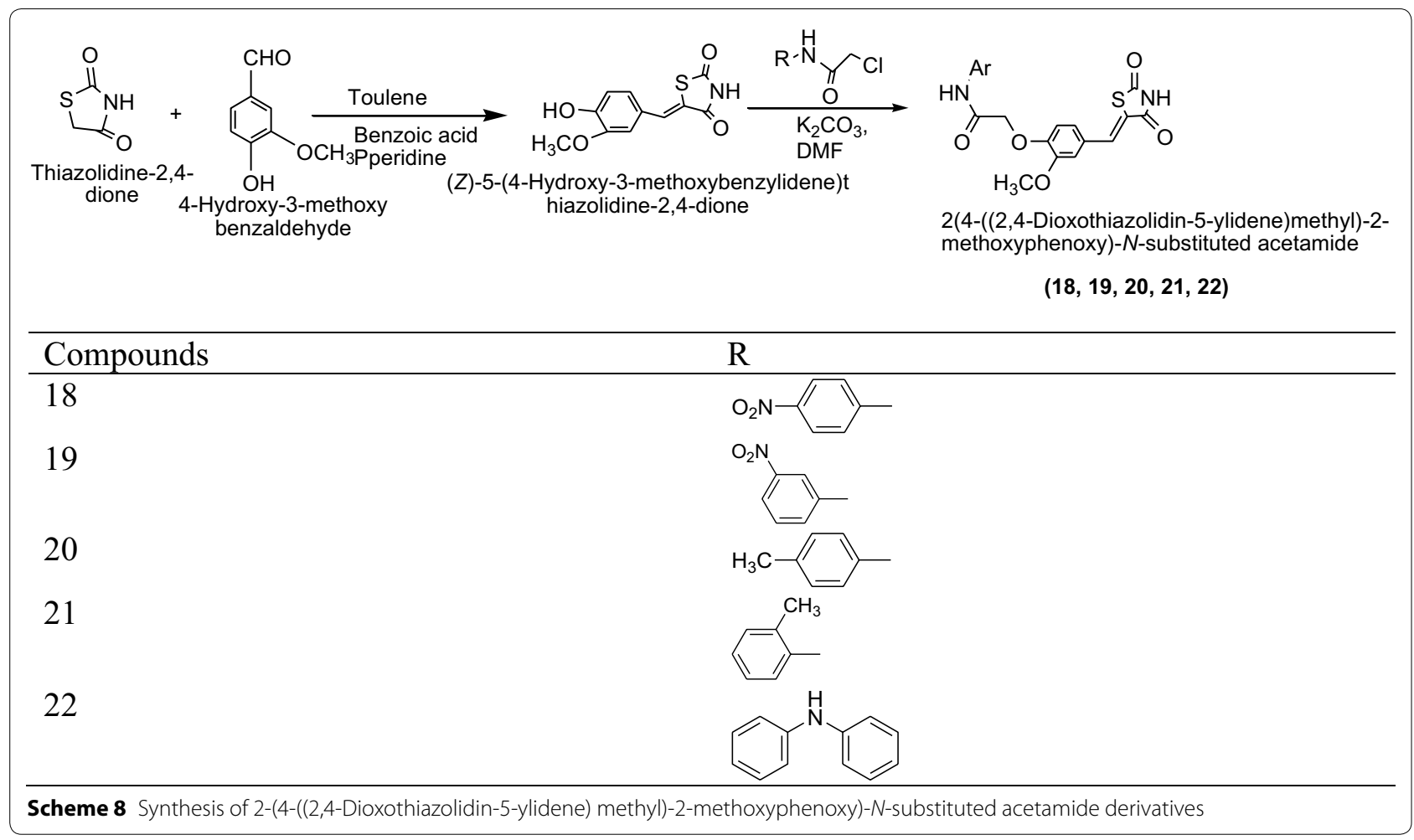

Table 8 Evaluation of hypoglycemic activity: effect of compound on \% decrease in blood glucose in diabetic mice

\begin{tabular}{|c|c|c|c|c|c|}
\hline Compounds & $\mathrm{Oh}$ & $2 \mathrm{~h}$ & $4 \mathrm{~h}$ & $6 \mathrm{~h}$ & $24 \mathrm{~h}$ \\
\hline Control & $252.53 \pm 4.254$ & $4.74 \pm 0.68$ & $7.9 \pm 4.32$ & $13.43 \pm 2.68$ & $3.18 \pm 4.35$ \\
\hline Piogiltazone & $250.75 \pm 5.21$ & $31.07 \pm 6.74$ & $37.48 \pm 5.37$ & $45.41 \pm 3.67$ & $10.3 \pm 6.53$ \\
\hline 18 & $252.79 \pm 2.85$ & $29.34 \pm 4.53$ & $36.52 \pm 5.43$ & $46.64 \pm 4.52$ & $6.70 \pm 6.51$ \\
\hline 19 & $252.19 \pm 4.35$ & $24.7 \pm 3.97$ & $34.76 \pm 6.51$ & $37.89 \pm 5.43$ & $5.19 \pm 7.74$ \\
\hline 20 & $254.38 \pm 4.53$ & $26.64 \pm 5.28$ & $34.26 \pm 5.67$ & $37.05 \pm 4.62$ & $4.19 \pm 5.43$ \\
\hline 21 & $253.60 \pm 5.64$ & $22.9 \pm 4.72$ & $35.6 \pm 5.53$ & $40.41 \pm 5.97$ & $3.87 \pm 6.53$ \\
\hline 22 & $252.73 \pm 5.23$ & $29.01 \pm 6.54$ & $36.47 \pm 4.65$ & $39.21 \pm 5.74$ & $3.0 \pm 3.75$ \\
\hline
\end{tabular}

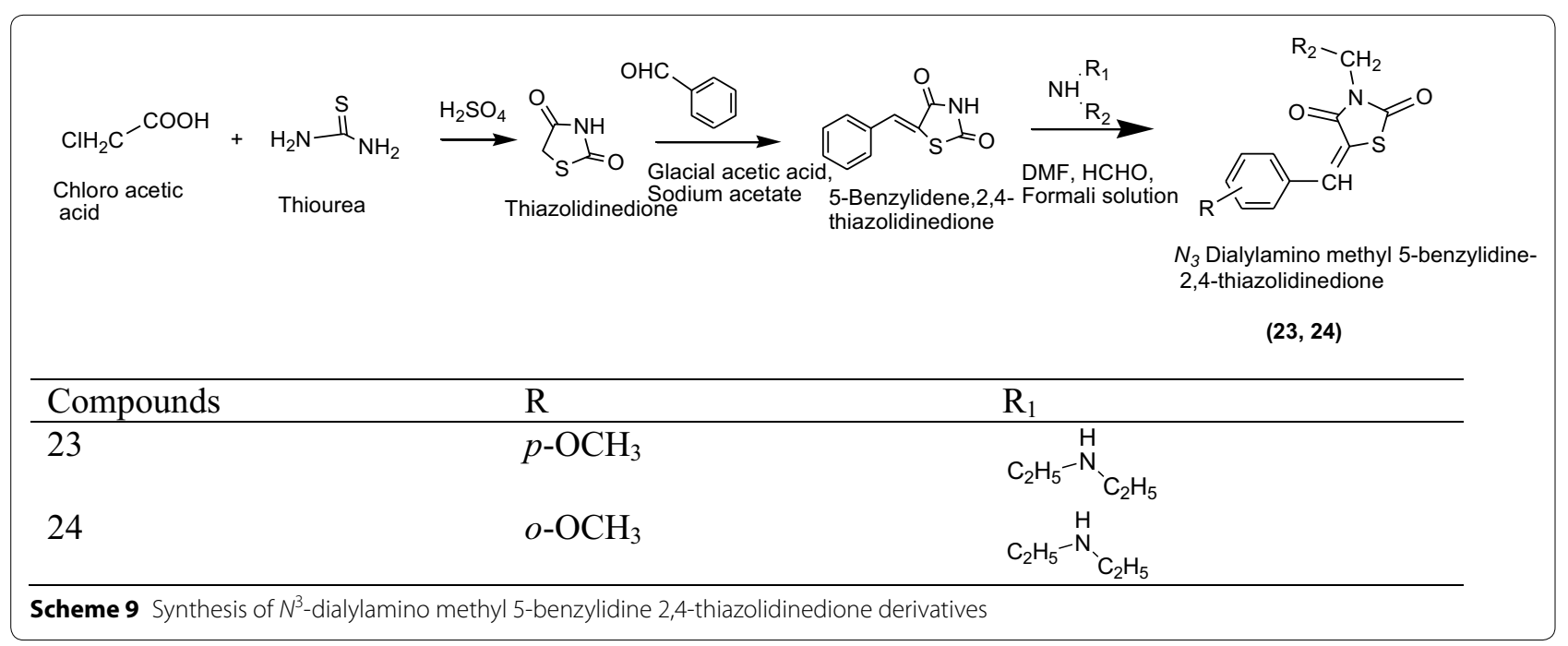


Table 9 Hypoglycemic activity of synthesized derivatives

\begin{tabular}{|c|c|c|c|c|c|c|}
\hline \multirow[t]{2}{*}{ Compounds } & \multirow[t]{2}{*}{ Dose (mg/kg) } & \multicolumn{3}{|c|}{ Mean blood glucose level (mg/dl) } & \multicolumn{2}{|c|}{$\begin{array}{l}\% \text { reduction in blood glu- } \\
\text { cose level }\end{array}$} \\
\hline & & Before 1st dose & After $2 \mathrm{~h}$ & After $4 \mathrm{~h}$ & After $2 \mathrm{~h}$ & After $4 \mathrm{~h}$ \\
\hline 23 & 50 & 400 & 56 & 48 & 86 & 88 \\
\hline 24 & 50 & 275 & 63 & 79 & 72 & 65 \\
\hline Rosiglitazone & 50 & 400 & 56 & 48 & 86 & 88 \\
\hline
\end{tabular}

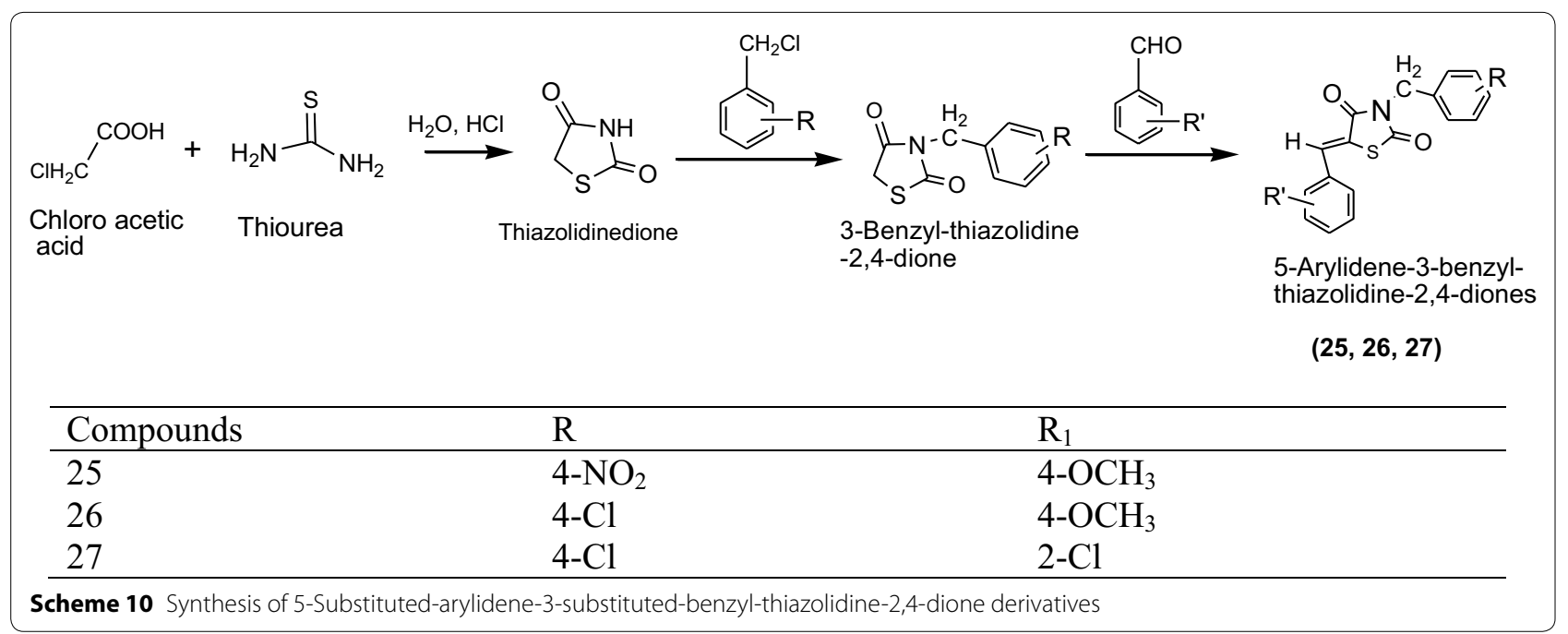

Table 10 Hypoglycemic activity of synthesized derivatives

\begin{tabular}{|c|c|c|c|c|}
\hline \multirow[t]{2}{*}{ Treatment (mg/kg) } & \multicolumn{4}{|c|}{ Blood glucose level (mg/dl) } \\
\hline & 0 day & 3rd day & 5th day & 7th day \\
\hline 25 & $86.11 \pm 0.98$ & $85.67 \pm 0.58$ & $84.68 \pm 0.54$ & $86.23 \pm 0.48$ \\
\hline 26 & $188.23 \pm 1.14$ & $189.56 \pm 0.98$ & $185 \pm 0.86$ & $182.36 \pm 1.25^{*}$ \\
\hline 27 & $189.35 \pm 1.18$ & $206.38 \pm 0.86$ & $192.30 \pm 1.2$ & $188.36 \pm 1.23$ \\
\hline Rosiglitazone & $194.99 \pm 1.70$ & $207.45 \pm 0.69$ & $189.64 \pm 1.33$ & $172.38 \pm 2.24$ \\
\hline
\end{tabular}

* indicates high reduction in glucose level after seven days

results of most active derivatives showed in Table 12 (Jawale et al. [16]).

\section{Thiazolidinedione derivatives as antimicrobial agents}

Long-ago, contagious diseases caused by multidrugresistant microorganisms have become a serious issue, representing a growing threat to human health and being a major problem in many countries worldwide. There has been a significant increase in clinical drug resistance over the past few decades, owing to exploitation of antimicrobial agents, thus many infectious disease can no longer be treated successfully with general anti-infective agents [17]. Modern therapies and management technique such as bone marrow or solid-organ transplants, and newer much aggressive chemotherapy have resulted in a rapidly inflating number of immune-suppressed patient. So, in order to meet above mentioned challenges, there is an urgent need for the development of novel antimicrobial agents [18].

In this study, Nawale et al. [19] synthesized a new series of 5-Substituted 2,4-thiazolidinedione derivatives (Scheme 13) and evaluated for in vitro antimicrobial 


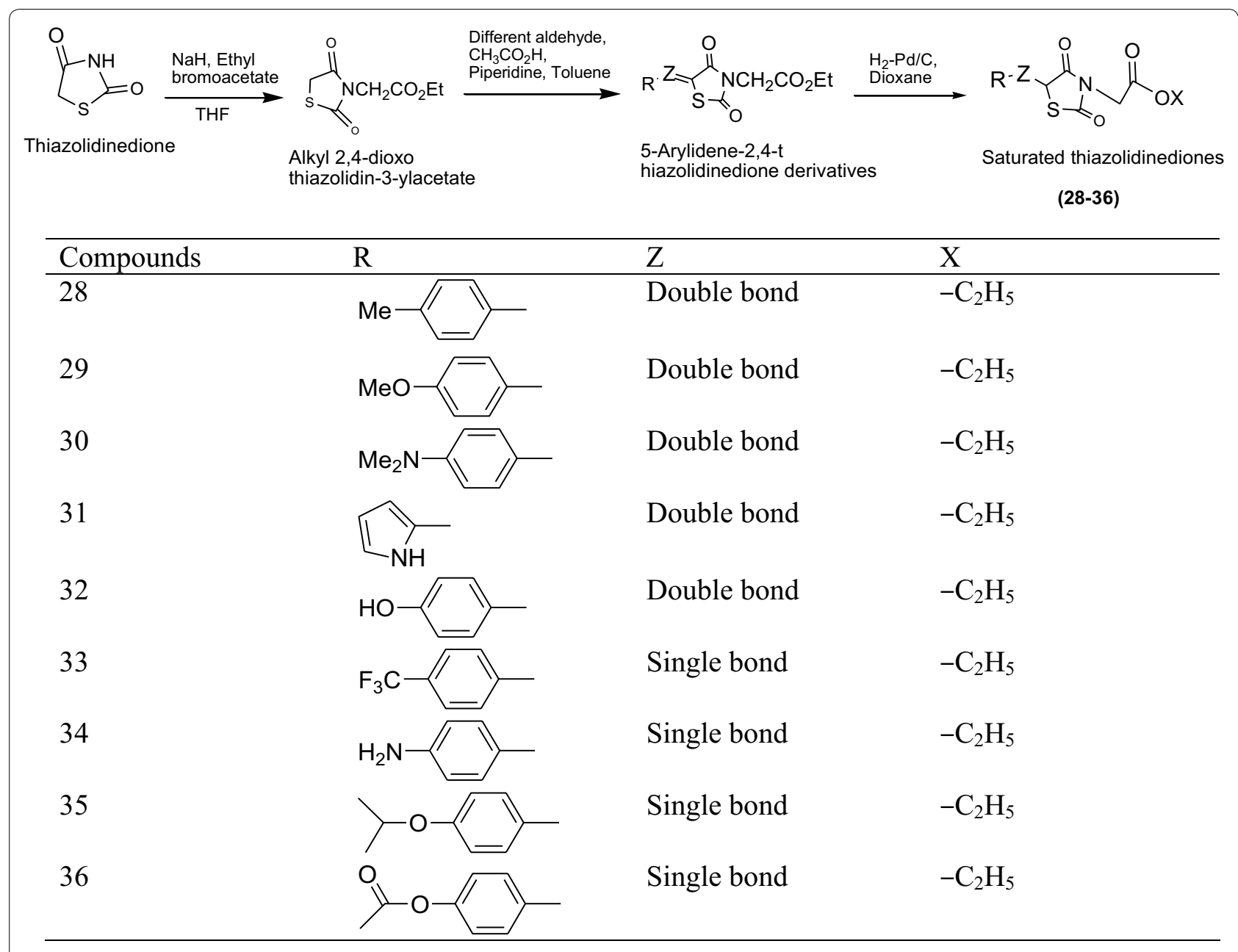

Scheme 11 Synthesis of [5-(4-Methoxy-benzylidene)-2,4-dioxo-thiazolidin-3-yl]-acetic acid

Table 11 Antihyperglycemic activity profile of title compounds thiazolidine-2,4-dione derivatives

\begin{tabular}{llll}
\hline Compounds & \multirow{2}{*}{$\begin{array}{l}\text { Antihyperglycemic activity, } \\
\text { SLM }\end{array}$} & \multicolumn{2}{l}{ PPARc } \\
\cline { 3 - 4 } & & $\mathbf{1 0} \mathbf{n m o l}$ & $\mathbf{1 0 0 0} \mathbf{n m o l}$ \\
\hline $\mathbf{2 8}$ & -22.1 & 9 & 9 \\
$\mathbf{2 9}$ & -22.2 & 7 & 8 \\
$\mathbf{3 0}$ & -15.8 & - & - \\
$\mathbf{3 1}$ & +9.00 & - & - \\
$\mathbf{3 2}$ & -26.7 & 10 & 12 \\
$\mathbf{3 3}$ & -12.3 & 9 & 11 \\
$\mathbf{3 4}$ & -12.7 & 8 & 10 \\
$\mathbf{3 5}$ & -4.1 & - & - \\
$\mathbf{3 6}$ & -26.8 & - & - \\
Rosiglitazone & 11.6 & 92 & 248 \\
Metformin & 34.1 & - & - \\
\hline
\end{tabular}

$P P A R_{c}$ proxisome proliferator activated receptor activity against two species of Gram-positive bacteria, Bacillus subtilis, Staphylococcus aureus and Gram-negative bacteria, Pseudomonas aeruginosa using broth dilution method. Among the synthesized derivatives, compounds 41, 42, 43 and 44 exhibited highest activity on all tested microorganisms. The results of synthesized compounds presented in Table 13 (Nawale et al. [19]).

Nastas et al. [20] synthesized a series of novel 5-(Chromene-3-yl)methylene-2,4-thiazolidinedione derivatives as presented in Scheme 14 and tested for its in vitro antimicrobial potency towards Gram-positive bacteria (Listeria monocytogenes, Staphylococcus aureus) and Gram-negative bacteria (Escherichia coli, Salmonella typhi) pathogenic bacteria and fungi (Candida albicans) using broth dilution method and the disk diffusion method. Among the synthesized derivatives, compounds 45, 46 and 47 antimicrobial activity against all tested 


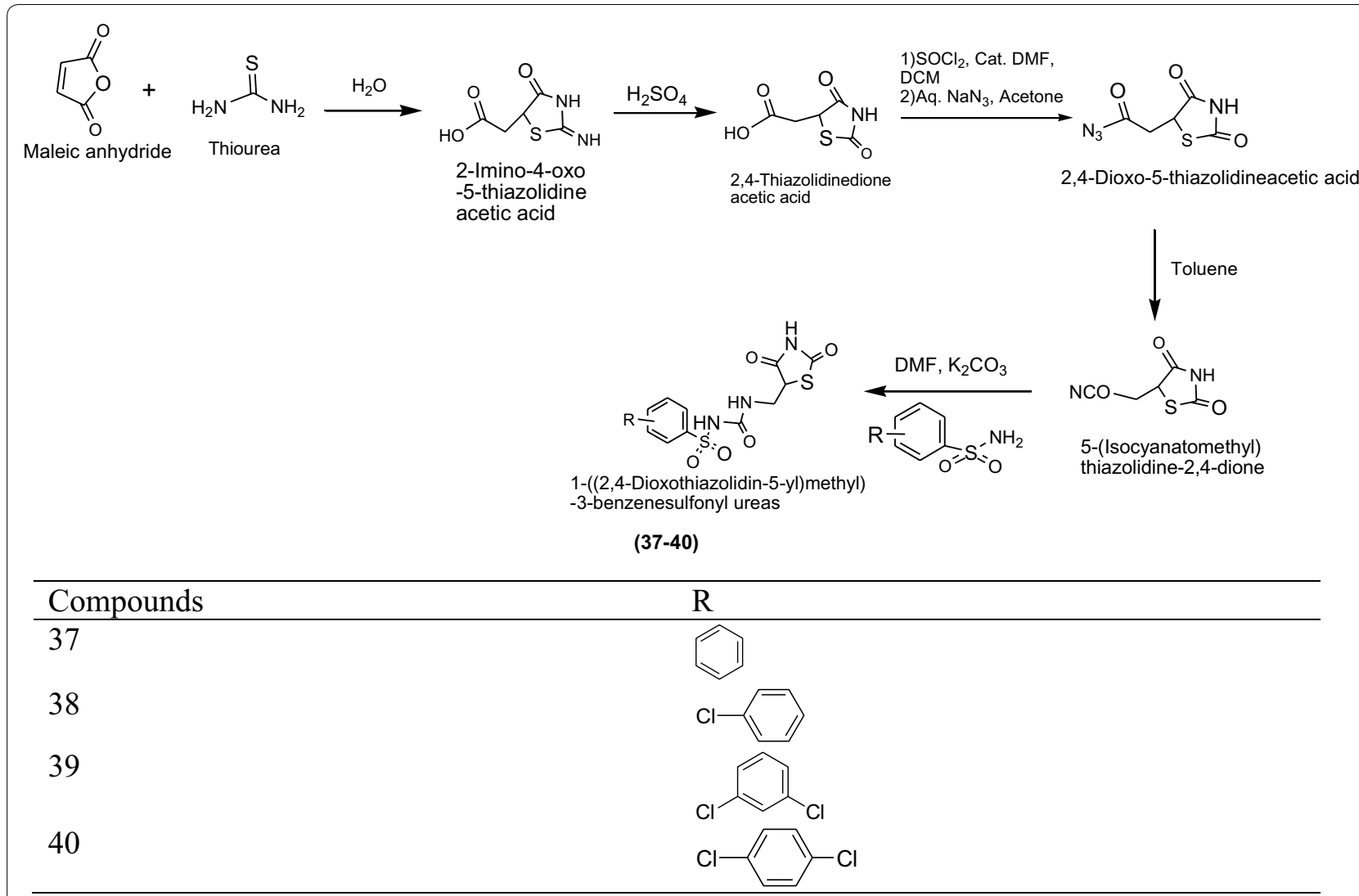

Scheme 12 Synthesis of 1-((2,4-Dioxothiazolidin-5-yl)methyl)-3-substitued benzene sulphonyl ureas

Table 12 Antidiabetic activity of synthesized compounds

\begin{tabular}{llll}
\hline Compounds & Dose $(\mathbf{m g} / \mathbf{d} \mathbf{l})$ & \% activity & Significance \\
\hline $\mathbf{3 7}$ & 100 & 15.8 & $\mathrm{p}<0.01$ \\
$\mathbf{3 8}$ & 100 & 17.2 & $\mathrm{p}<0.01$ \\
$\mathbf{3 9}$ & 100 & 14.3 & $\mathrm{p}<0.05$ \\
$\mathbf{4 0}$ & 100 & 16.5 & $\mathrm{p}<0.01$ \\
Metformin & 100 & 27.0 & $\mathrm{p}<0.001$ \\
\hline
\end{tabular}

bacteria and fungi. The results of most active derivatives showed in Table 14 (Nastas et al. [20]).

Moorthy et al. [5] synthesized a series of novel imidazolyl thiazolidinedione derivatives (Scheme 15) and screened them for their in vitro antimicrobial activity towards Gram-positive (S. aureus, S. epidermidis, $M$. luteus, B. cereus) and Gram-negative (E. coli, P. aeruginosa, K. pneumonia) bacteria and fungi (A.niger, $A$. fumigates). They were compared with standard drug ciprofloxacin and ketoconazole. Among the synthesized derivatives, compound 48 [Methyl-2-(4-((3-(2-methoxy2-oxoethyl)-2,4-dioxothiazolidin-5-ylidene)methyl) $1 \mathrm{H}$ Imidazol-1-yl)acetate] showed potent activities towards $S$. aureus, $S$. epidermidis, E. coli, P. aeruginosa, A. niger and $A$, fumigates and 49 [Methyl-2-(2-((2,4-dioxothiazolidin-5-ylidene)methyl)- $1 H$-imidazol-1-yl)acetate], 50 [Methyl-2-(2-((3-(2-methoxy-2-oxoethyl)2,4-dioxothiazolidin-5-yldiene)methyl) $1 H$-imidazol-1-yl)acetate] and 51 [5-(4-Bromobenzylidene)thiazolidine-2,4-dione] showed good activity against all microorganism. The results of synthesized compounds presented in Table 15 (Moorthy et al. [5]).

Alagawadi et al. [21] designed some novel derivatives of imidazole fused with thiazolidine-2,4-dione and evaluated them for their antibacterial activity against 


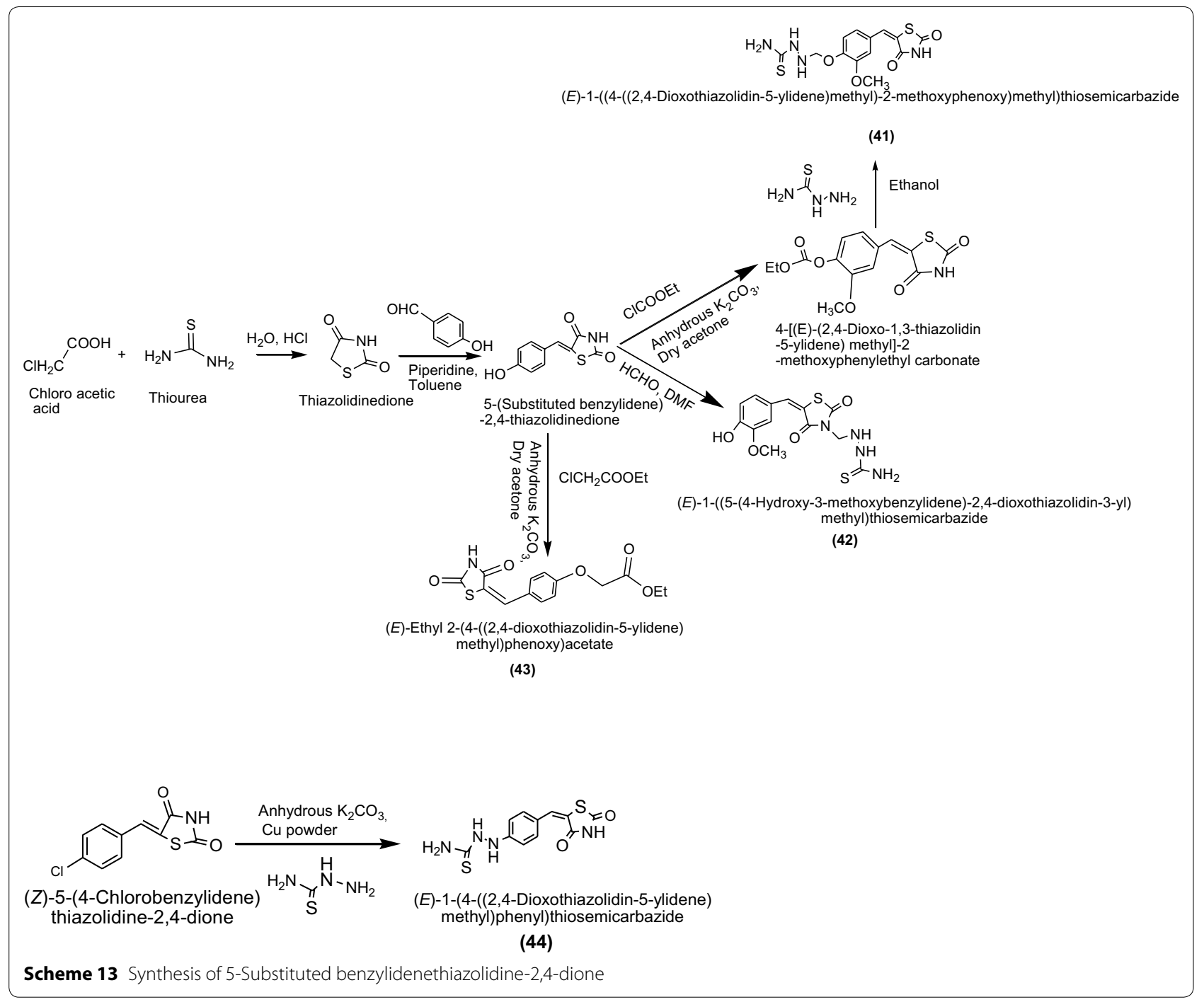

Table 13 MIC $(\mu \mathrm{g} / \mathrm{ml})$ values for the screened thiazolidinediones compounds

\begin{tabular}{llll}
\hline Compounds & \multicolumn{2}{l}{ Microorganisms } \\
\cline { 2 - 4 } & Bacillus subtilis & $\begin{array}{l}\text { Staphylococcus } \\
\text { aureus }\end{array}$ & $\begin{array}{l}\text { Pseudomonas } \\
\text { aeruginosa }\end{array}$ \\
\hline $\mathbf{4 1}$ & 31.25 & 31.25 & 31.25 \\
$\mathbf{4 2}$ & 31.25 & 31.25 & 31.25 \\
$\mathbf{4 3}$ & 62.5 & 125 & 62.5 \\
$\mathbf{4 4}$ & 31.25 & 62.5 & 125 \\
Streptomycin & 3.90 & 3.90 & 3.90 \\
\hline
\end{tabular}

Gram-positive bacteria Staphylococcus aureus (S. a), Enterococcus faecalis (E. f) Gram-negative bacteria Escherichia coli (E. c.) Pseudomonas aeruginosa (P. a.) and antifungal activity Candida albicans (C.a.) Cryptococcus neoformans (C. n.) Aspergillus flavus (A.f.) and Aspergillus niger (A.n.) Among the screened compound the MIC value of compound 52 [5-\{[2-(3,4,5-trimethoxyphenyl)6-(4-bromophenyl)imidazo[2,1-b][1,3,4] thiadiazol-5-yl] methylidene\}-1,3-thiazolidine-2,4-dione], 53 [5-\{[2-(3,4, 5-trimethoxyphenyl)-6-(4-chlorophenyl)midazo[1-b] $[1,3,4]$ thiadiazol-5-yl] methylidene $\}$-1,3-thiazolidine2,4-dione $]$ (Scheme 16), $54 \quad[N-[-($ dimethylamino) 


6,8-Dichloro-4-oxo-4H-
chromene-3-carbaldehyde

Table 14 Antimicrobial activity of 5-(chromene-3-yl)methylene-2,4-thiazolidinediones

\begin{tabular}{|c|c|c|c|c|c|}
\hline \multirow{2}{*}{$\begin{array}{l}C_{\mathrm{p}} \\
10 / 5 / 1(\mathrm{mg} / \\
\mathrm{ml})\end{array}$} & \multicolumn{2}{|c|}{ Gram-positive } & \multicolumn{2}{|c|}{ Gram-negative } & \multirow{2}{*}{$\begin{array}{l}\text { Fungi } \\
\text { C.albicans }\end{array}$} \\
\hline & $\begin{array}{l}\text { L. mono- } \\
\text { cytogenes }\end{array}$ & S. aureus & E. coli & S. typhi & \\
\hline 45 & $18 / 22 / 18$ & $22 / 12 / 12$ & $12 / 14 / 14$ & $15 / 19 / 20$ & 20/18/18 \\
\hline 46 & $22 / 22 / 20$ & $24 / 28 / 28$ & 18/18/16 & 20/18/16 & 18/18/16 \\
\hline 47 & $28 / 28 / 28$ & $28 / 28 / 28$ & 18/18/18 & 18/18/18 & $22 / 22 / 22$ \\
\hline Gentamicin & 18 & 19 & 22 & 18 & NT \\
\hline Fluconazole & NT & NT & NT & NT & 28 \\
\hline
\end{tabular}

NT not tested methylidene $]-5$-[(2,4-dioxo-1,3-thiazolidin-5-ylidene) methyl]-6-phenylimidazo[2,1-b][1, 3, 4]thiazolie-2-sulfonamide] and 55 [ $N$-[-(dimethylamino)methylidene]5-[-(2,4-dioxo-1,3-thiazolidin-5-ylidene)methyl]-6-(4bromophenyl)-imidazo[2,1-b][1,3,4] thiazole-2-sulfonamide] (Scheme 17) were showed potent activity against Gram-positive, Gram-negative bacterial strain and fungal strains. The significant results of these compounds are presented in Table 16 (Alagawadi et al. [21]).

Khan et al. [22] designed some novel biphenyl tetrazole thiazolidinedione derivatives (Scheme 18) and evaluated for their antimicrobial activity against bacterial strain 


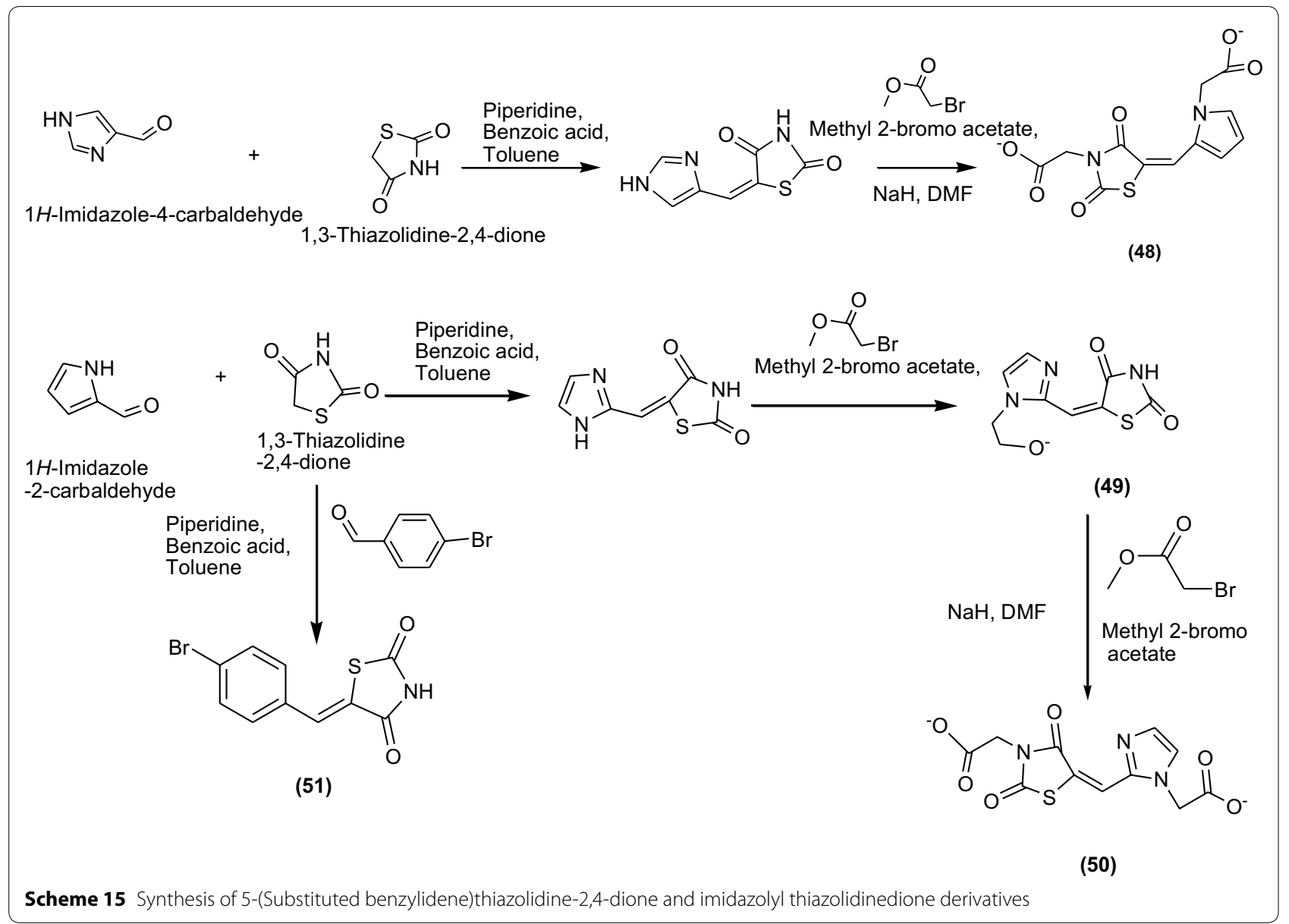

Table 15 In vitro activity zone of inhibition $(\mathrm{mm})$ of compounds

\begin{tabular}{|c|c|c|c|c|c|c|}
\hline \multirow[t]{2}{*}{ Compounds } & \multicolumn{2}{|c|}{ Gram-positive bacteria } & \multicolumn{2}{|c|}{ Gram-negative bacteria } & \multicolumn{2}{|l|}{ Fungi } \\
\hline & S. aureus & S. epidermidis & E. coli & P. aeruginosa & A. niger & A. fumigates \\
\hline 48 & $18(1.9)$ & $16(1.4)$ & $28(1.6)$ & $28(0.56)$ & $20(8.8)$ & $26(2.3)$ \\
\hline 49 & $21(22.1)$ & $27(22.2)$ & $27(21.5)$ & $21(21.5)$ & $24(20.7)$ & $20(22.6)$ \\
\hline 50 & $16(2.7)$ & $18(3.39)$ & $22(9.2)$ & $16(1.4)$ & $22(8.2)$ & $26(3.4)$ \\
\hline 51 & $21(22.1)$ & $25(22.2)$ & $25(21.5)$ & $21(21.5)$ & $28(21.6)$ & $25(21.7)$ \\
\hline Ciprofloxacin & $29(0.2)$ & $31(0.39)$ & $32(0.2)$ & $33(0.25)$ & - & - \\
\hline Ketoconazole & - & - & - & - & $26(6.1)$ & $24(0.23)$ \\
\hline
\end{tabular}

(Escherichia coli, Bacillus subtilis). Antimicrobial activity result indicated that among the synthesized derivatives $56[(E)-3-((20-(1 H)$-tetrazol-5-yl)biphenyl-4-yl)methyl)5-(4-chlorobenzylidene)thiazolidine-2,4-dione],
((E)-3-((20-(1H-tetrazol-5-y)biphenyl-4-yl)methyl)-5-(2chlorophenylbenzylidene)thiazolidine-2,4-dione) and 58 [(E)-3-((20-(1H-tetrazol-5-yl)biphenyl-4-yl)methyl)5-(2,6-dichlorobenzylidene) thiazolidine-2,4-dione] 


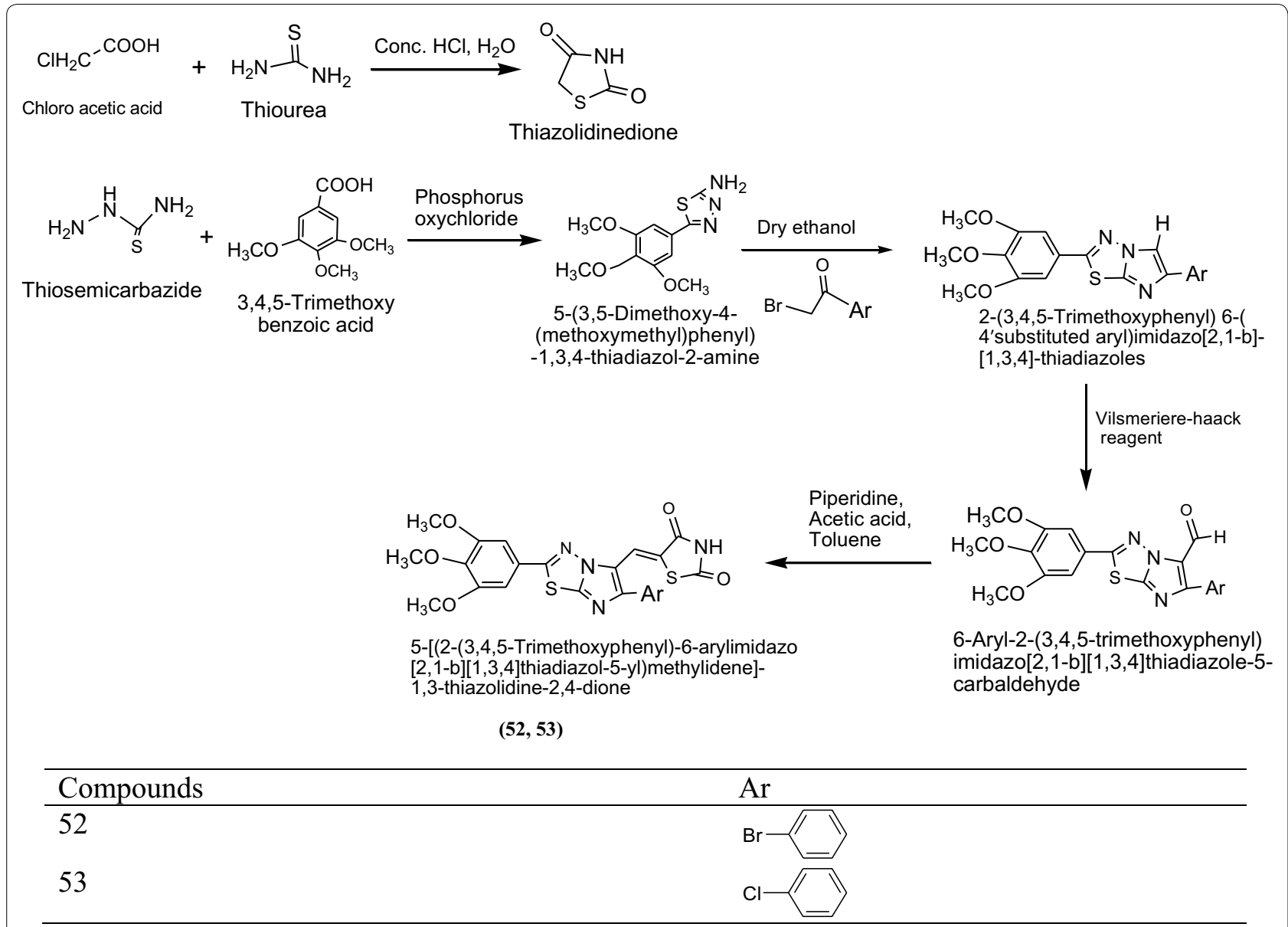

Scheme 16 Synthesis of 5-[(2-(3,4,5-Trimethoxyphenyl)-6-arylimidazo[2,1-b][1,3,4]thiadiazol-5-yl)methylidene]-1,3-thiazolidine-2,4-dione

showed potent in vitro antimicrobial activity. The results of most active derivatives showed in Table 17 (Khan et al. [22]).

Liu et al. [23] synthesized a series of new compound bearing 2,4-thiazolidinedione and benzoic moiety as presented in Scheme 19 and screened for their in vitro antimicrobial activity against bacterial strain (Staphylococcus aureus and Escherichia coli). Antimicrobial activity result indicated that among the synthesized derivatives, compounds 59, 60, 61, 62 and 63 showed highest in vitro growth of inhibition against bacterial strains. The results of synthesized compounds presented in Table 18 (Liu et al. [23]).
Purohit et al. [24] synthesized a series of novel 3,5-disubstituted thiazolidinediones derivatives (Scheme 20) and evaluated its antibacterial activity against Staphylococcus aureus, Enterococcus faecalis, Klebsiella pneumonia, Escherichia coli and antifungal activity was performed against Candia albicans, Aspergillus niger, Aspergillus flavus. The screening results were compared with ciprofloxacin, norfloxacin for antibacterial and fluconazole, griseofulvin for antifungal activity respectively. Among the synthesized compounds 64, 65, 66 and 67 showed highest antimicrobial potency and their structure were. The significant results of these compounds are presented in Table 19 (Purohit et al. [24]). 


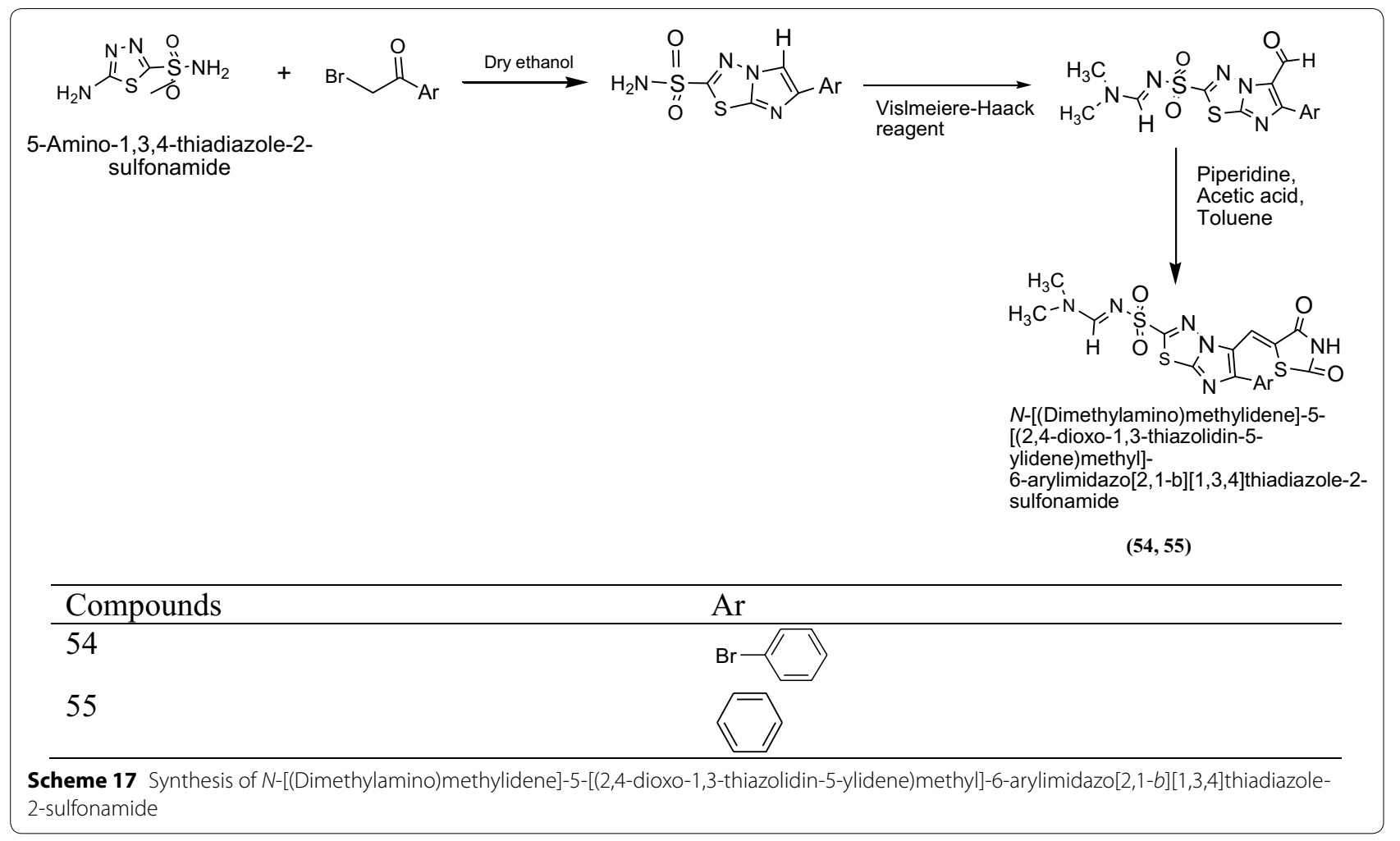

Table 16 Antimicrobial activities of synthesized compounds

\begin{tabular}{|c|c|c|c|c|c|c|c|c|}
\hline \multirow[t]{2}{*}{ Compounds } & \multicolumn{8}{|c|}{ Minimum inhibitory concentration (MIC) in $\mu \mathrm{g} / \mathrm{ml}$} \\
\hline & E.c & P. $a$ & S. $a$ & E.f & C. $a$ & C. $n$ & A.f & A. $n$ \\
\hline 52 & 256 & 256 & 32 & 32 & 4 & 8 & 4 & 4 \\
\hline 53 & 128 & 64 & 32 & 32 & 4 & 8 & 32 & 32 \\
\hline 54 & 128 & 32 & 8 & 4 & 1 & 2 & 4 & 4 \\
\hline 55 & 64 & 64 & 8 & 8 & 4 & 8 & 4 & 4 \\
\hline Ampicillin & 2 & 2 & 1 & 2 & - & - & - & - \\
\hline Ketoconazole & - & - & - & - & 2 & 1 & 2 & 1 \\
\hline
\end{tabular}

Sharma et al. [25] synthesized a series of novel $\mathrm{N}$-(-5-arylidene-2-(4-chlorophenyl)-4-oxothiazolidin-3-yl) isonicotnamide derivatives by knoevenagel condensation using Scheme 21 and assayed for antibacterial activity against Escherichia coli, Staphylococcus aureus, Bacillus subtilis and antifungal activity against Candida albicans, Aspergillus niger, Saccharomyces cervesia using turbidimetric method. Among the synthesized compounds $\mathbf{6 8}$ 


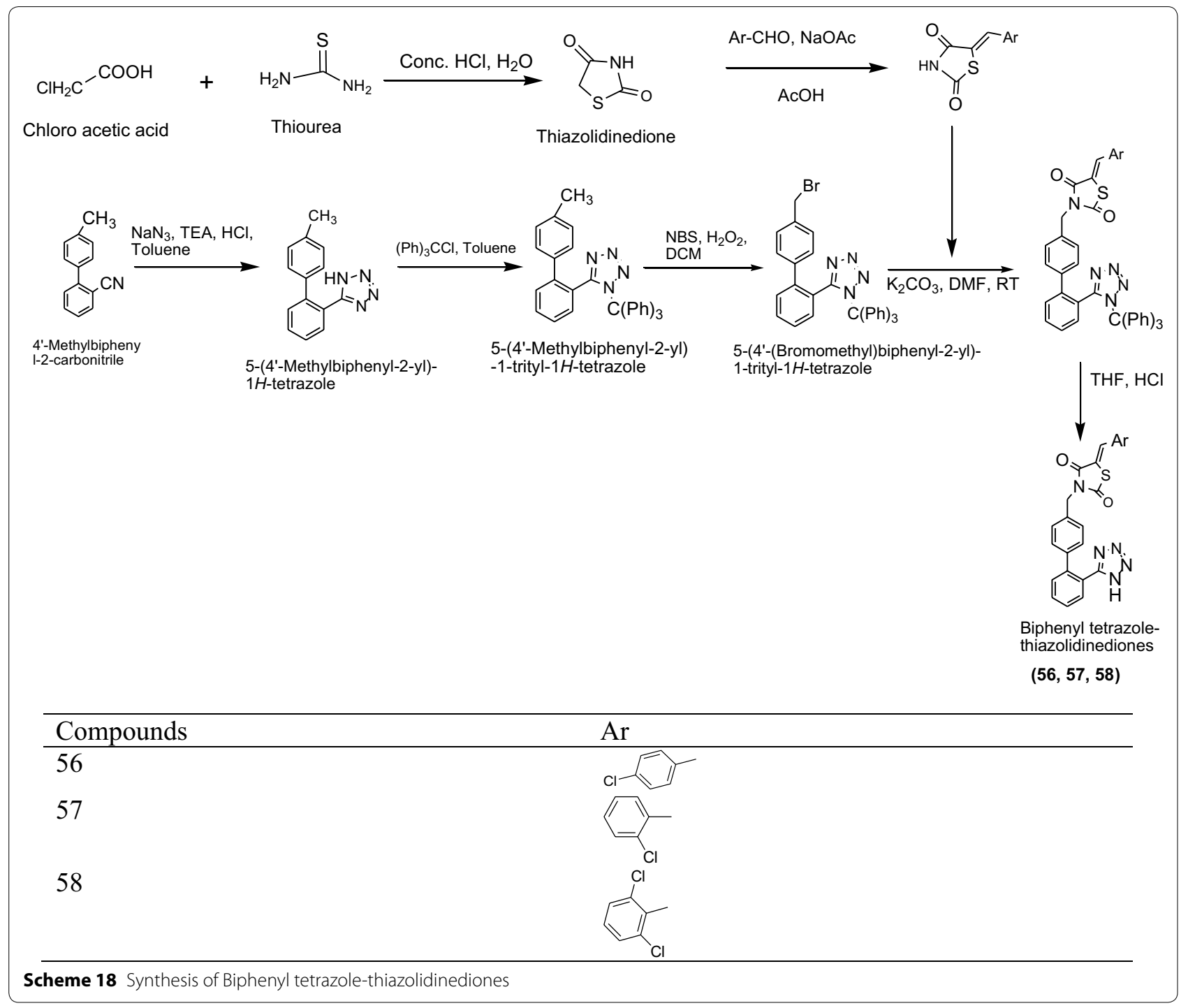

Table 17 Antibacterial activities of synthesized compounds

\begin{tabular}{llr}
\hline Compounds & \multicolumn{2}{l}{ MIC \pm SLM $(\boldsymbol{\mu g} / \mathbf{m l})$} \\
\cline { 2 - 3 } & \multicolumn{1}{c}{ E. coli } & \multicolumn{1}{c}{ B. subtilis } \\
\hline $\mathbf{5 6}$ & $20.75 \pm 1.55$ & $35.41 \pm 2.41$ \\
$\mathbf{5 7}$ & $19.41 \pm 1.27$ & $26.00 \pm 1.96$ \\
$\mathbf{5 8}$ & $8.58 \pm 0.42$ & $8.42 \pm 0.51$ \\
Ciprofloxacin & $25.00 \pm 0.95$ & $50.00 \pm 1.75$ \\
\hline
\end{tabular}

(N-(5-benzylidene-2-(4-chlorophenyl)-4-oxothiazolidin3-yl)isonicotinamide), 69 (N-(2-(4-chlorophenyl)-5-(furan2-ylmethylene)-4-oxothiazolidin-3-yl)isonicotinamide) and 70 (N-(5-(2-nitrobenzylidene)-2-(4-chlorophenyl)-4-oxothiazolidin-3-yl)isonicotinamide) result in wide spectrum antimicrobial activity against all the test bacteria and fungi using ciprofloxacin and clotrimazole as a standard drug respectively. The results of synthesized compounds presented in Table 20 (Sharma et al. [25]). 


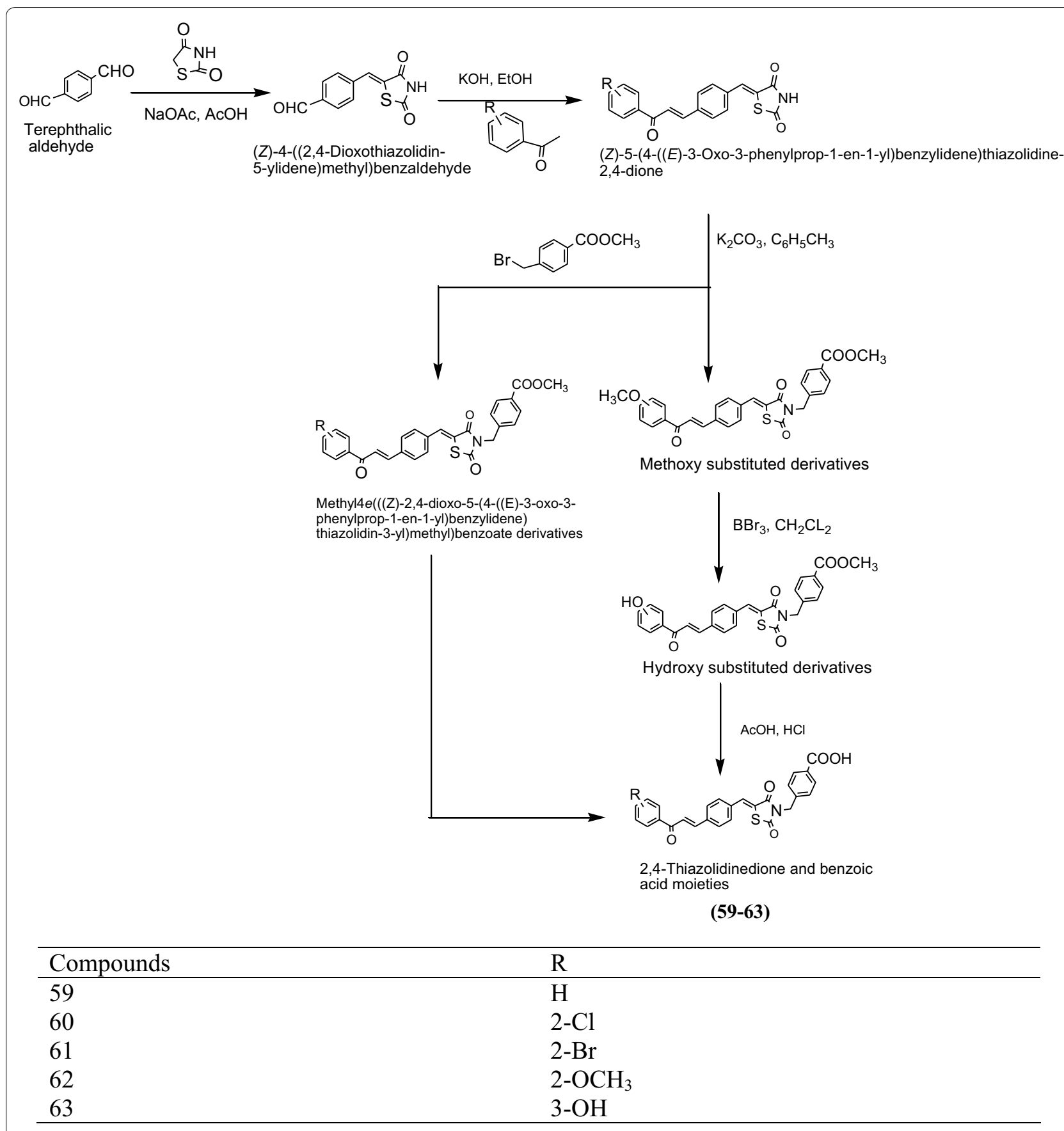

Scheme 19 Synthesis of 4-(((Z)-5-((4-((E)-3-(Substituted)-3-oxoprop-1-en-1-yl)benzylidene)-2,4-dioxothiazolidin-3-yl)methyl)benzoic acid 
Table 18 Inhibitory activities of novel compounds against bacteria

\begin{tabular}{llllll}
\hline Compounds & \multicolumn{2}{l}{ S.aureus } & & & \multicolumn{2}{l}{ E. coli } & \\
\cline { 2 - 3 } & $\mathbf{4 2 2 0}$ & $\mathbf{5 3 0}$ & & $\mathbf{1 3 5 6}$ & $\mathbf{1 6 8 2}$ \\
\hline $\mathbf{5 9}$ & 1 & 2 & $>64$ & $>64$ \\
$\mathbf{6 0}$ & 1 & 2 & $>64$ & $>64$ \\
$\mathbf{6 1}$ & 2 & 4 & $>64$ & $>64$ \\
$\mathbf{6 2}$ & 2 & 4 & $>64$ & $>64$ \\
$\mathbf{6 3}$ & 2 & 4 & $>64$ & $>64$ \\
Norfloxacin & 2 & 2 & 16 & 16 \\
Oxacillin & 1 & 1 & $>64$ & $>64$ \\
\hline
\end{tabular}

Thiazolidine-2,4-dione derivatives as anti-inflammatory agents

The future of anti-inflammatory compound lies in the development of orally active drugs that decreases production or activities of pro-inflammatory cytokines. Anti-inflammatory compounds are normally used for curing of different infectious conditions. Therefore, the rate of incidence of disease limits its clinical use. Thus here is requirement of designing advance drugs with improved activity and long term relieve from chronic inflammatory condition [26]. The complete knowledge and understanding of the pivotal role of inflammation in seemingly untreated diseases has resulted in development of novel anti-inflammatory agents [27].

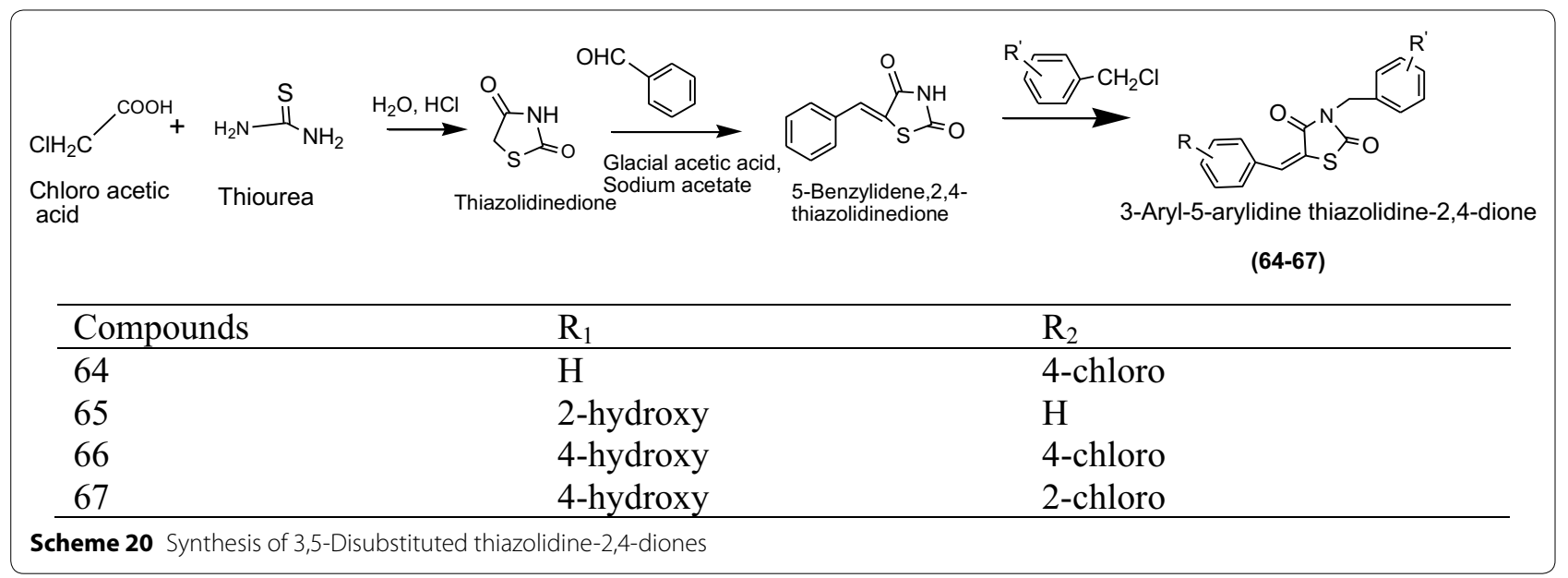

Table 19 Antimicrobial activities of synthesized compounds

\begin{tabular}{|c|c|c|c|c|c|c|c|}
\hline \multirow[t]{2}{*}{ Compounds } & \multicolumn{7}{|c|}{ Minimum inhibitory concentration (MIC $\mu \mathrm{g} / \mathrm{ml}$ ) } \\
\hline & S. aureus & E. faecalis & K. pneumonia & E. coli & C. albicans & A. niger & A. flavus \\
\hline 64 & 4 & 4 & 250 & 500 & 16 & 16 & 8 \\
\hline 65 & 4 & 31.25 & 62.5 & 62.5 & 31.5 & 1 & 8 \\
\hline 66 & 2 & 4 & $>500$ & $>500$ & 4 & 8 & 8 \\
\hline 67 & 1 & 1 & 62.5 & 62.5 & 4 & 4 & 2 \\
\hline Ciprofloxacin & 2 & 2 & 1 & 2 & - & - & - \\
\hline Norfloxacin & 10 & 3.1 & 0.1 & 10 & - & - & - \\
\hline Fluconazole & - & - & - & - & 16 & 8 & 8 \\
\hline Griseofulvin & - & - & - & - & 500 & 100 & 7.5 \\
\hline
\end{tabular}




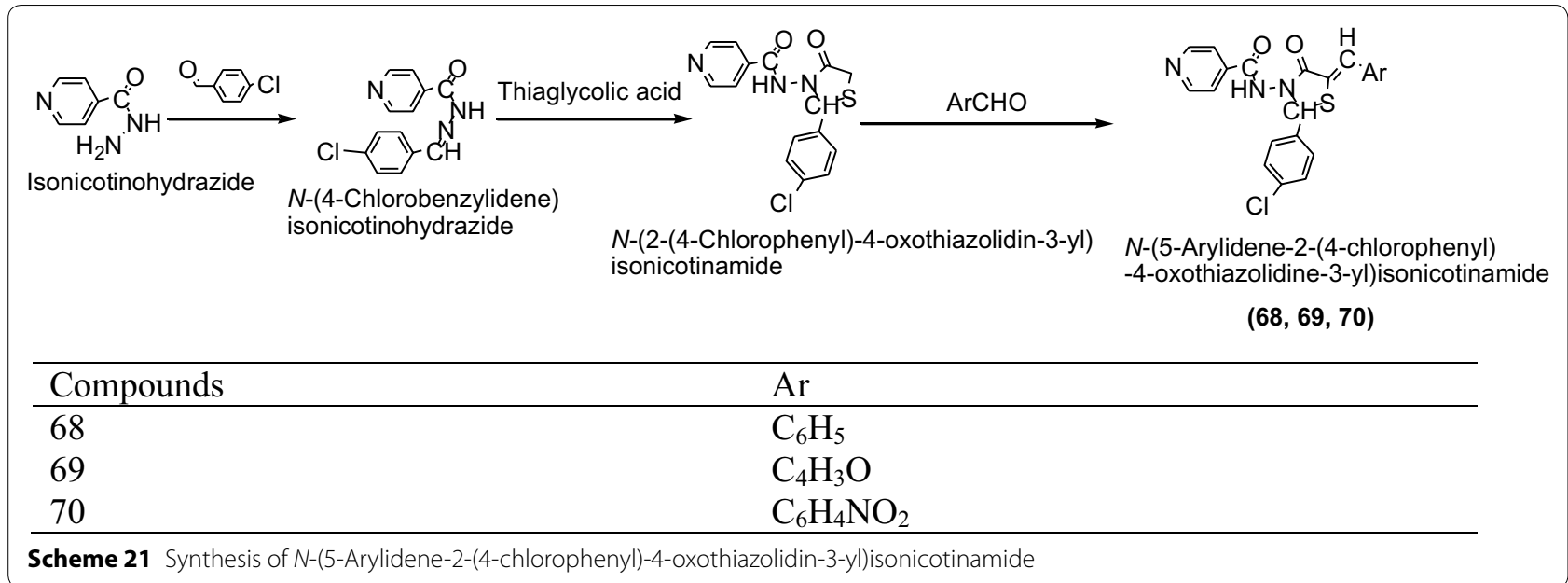

Table 20 Antimicrobial activities of synthesized compounds

\begin{tabular}{lllllll}
\hline Compounds & \multicolumn{2}{l}{ Minimum inhibitory concentration $(\mathbf{M I C})$ in $\boldsymbol{\mu g} / \mathbf{m l}$} \\
\cline { 2 - 7 } & E. coli & B. subtilis & S. aureus & A. niger & C. albicans & S. cerevisiae \\
\hline $\mathbf{6 8}$ & 1.25 & 1.25 & 0.62 & 0.62 & 0.31 & 1.25 \\
$\mathbf{6 9}$ & 0.62 & 0.31 & 0.62 & 0.62 & 0.15 & 0.62 \\
$\mathbf{7 0}$ & 0.31 & 0.62 & 0.31 & 0.62 & 0.15 & 0.31 \\
Ciprofloxacin & 0.15 & 0.25 & 0.01 & - & - & - \\
Clotrimazole & - & - & - & 0.10 & 0.30 & 0.20 \\
\hline
\end{tabular}

Youssef et al. [26] synthesized some novel active pyrazolyl-2,4-thiazolidinedione derivatives (Scheme 22) followed by their in vitro anti-inflammatory evaluation. Among them, compounds 71 and 72 [(Z)-3-allyl-5-((3(4-chlorophenyl)-1-phenyl- $1 H$-pyrazol-4-yl(methylene) thiazolidine-2,4-dione] showed moderate to good antiinflammatory activity using celecoxib as standard and turpentine oil as control. The results of potent derivatives presented in Tables 21, 22 and 23 (Youssef et al. [26]).

Ma et al. [28] synthesized a series of novel 5-benzylidene thiazolidine-2,4-dione derivatives as presented in Scheme 23 and screened for in vitro inflammation reduction activity. Among the synthesized derivatives, compounds 73 [(Z)-2-(4-((2,4-dioxothiazolidin-5-ylidene)methyl) phenoxy)- $N$-(3-fluorophenyl)acetamide $], \quad 74 \quad[(Z)-N-(3-$ chlorophenyl)-2-(4-((2,4-dioxothiazolidin-5-ylidene) methyl)phenoxy)acetamide $]$ and 75 [(Z)-2-(4-((2,4-dioxothiazolidin-5-ylidene)methyl)phenoxy)- $N$-(naphthalene1-yl)acetamide] were found to be most active anti-inflammatory agent compared to indomethacin as the standard. The results of potent compounds are accessible in Table 24 (Ma et al. [28]).

\section{Thiazolidinedione derivatives as anticancer agents}

Cancer is a genetic disorder that has always been a major threat all over the world and has been characterized by proliferation of abnormal cells and exhibiting an increasing mortality rate globally and being characterized by 


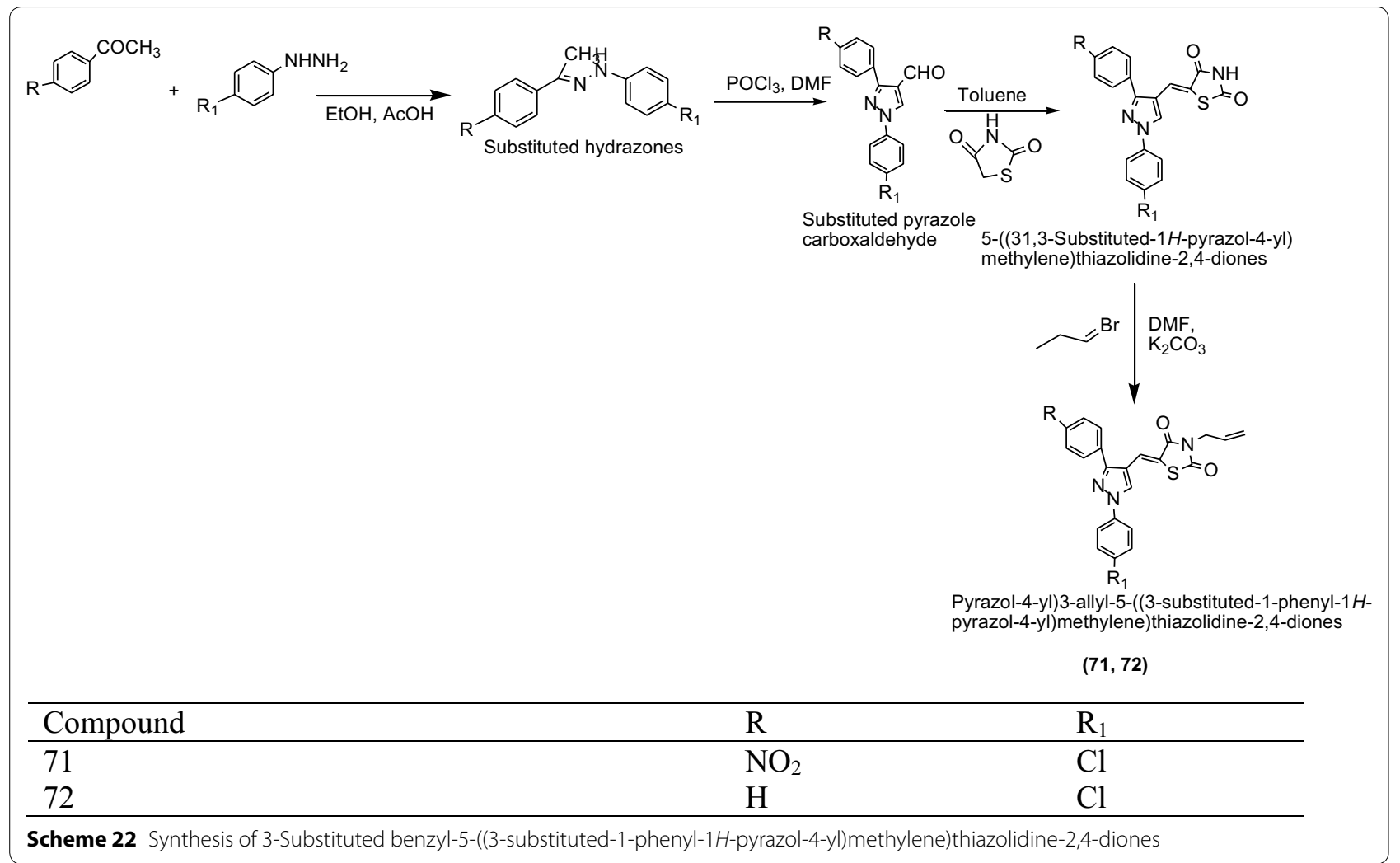

Table 21 Cyclooxygenase inhibition activity of synthesized compound

\begin{tabular}{llll}
\hline Compounds & Concentration (Um) (no. of experiments) & COX-1 activity (\% inhibition) & COX-2 activity (\% inhibition) \\
\hline $\mathbf{7 1}$ & $10(3)$ & $28.4 \pm 11.6$ & $19.4 \pm 8.2$ \\
$\mathbf{7 2}$ & $10(3)$ & $26.5 \pm 6$ & $13.6 \pm 1.1$ \\
Celecoxib & $10(3)$ & $0.3 \pm 2.5$ & $30.8 \pm 5.9$ \\
\hline
\end{tabular}

Table 22 Inflammation reduction results of synthesized compounds in Formalin induced rat paw edema bioassay

\begin{tabular}{llllll}
\hline Compounds & \multicolumn{3}{l}{ Volume of edema $(\mathbf{m l})$} & & \\
\cline { 2 - 6 } & $\mathbf{0} \mathbf{h}$ & $\mathbf{1} \mathbf{h}$ & $\mathbf{2}$ & $\mathbf{3} \mathbf{h}$ & $\mathbf{4 h}$ \\
\hline $\mathbf{7 1}$ & $0.31 \pm 0.001$ & $0.44 \pm 0.01(24)$ & $0.44 \pm 0.01(46)$ & $0.46 \pm 0.003(68)$ & $0.46 \pm 0.02(68)$ \\
$\mathbf{7 2}$ & $0.33 \pm 0.02$ & $0.41 \pm 0.01(53)$ & $0.42 \pm 0.01(63)$ & $0.46 \pm 0.01(72)$ & $0.49 \pm 0.01(66)$ \\
Control & $031 \pm 0.01$ & $0.40 \pm 0.01$ & $0.55 \pm 0.01$ & $0.78 \pm 0.01$ & $0.78 \pm 0.008$ \\
Celecoxib & $0.31 \pm 0.01$ & $0.41 \pm 0.005(41)$ & $0.43 \pm 0.02(50)$ & $0.50 \pm 0.005(60)$ & $0.48 \pm 0.03(68)$ \\
\hline
\end{tabular}

Table 23 Inflammation reduction results of synthesized compounds in turpentine oil induced granuloma pouch bioassay in rat

\begin{tabular}{lll}
\hline Compounds & Volume of exudates $(\mathbf{m l})$ & \% inhibition \\
\hline $\mathbf{7 1}$ & $1.12 \pm 0.06$ & 51 \\
$\mathbf{7 2}$ & $1.12 \pm 0.06$ & 50 \\
Control & $2.28 \pm 0.07$ & - \\
Celecoxib & $1.05 \pm 0.10$ & 54 \\
\hline
\end{tabular}

rapid formation of abnormal cells and spreading through metastasis to different organs [29, 30]. Currently available treatment (chemotherapy and radiotherapy) for most types of cancer only provide temporary therapeutic benefits as well as being limited by a narrow therapeutic index, remarkable toxicity and acquired resistance [31]. In recent times, advance in clinical researches for anticancer agents have been increased and as neoplastic cells are the anomalous proliferation of cells in the body which cause cancer, various effective compounds derived 


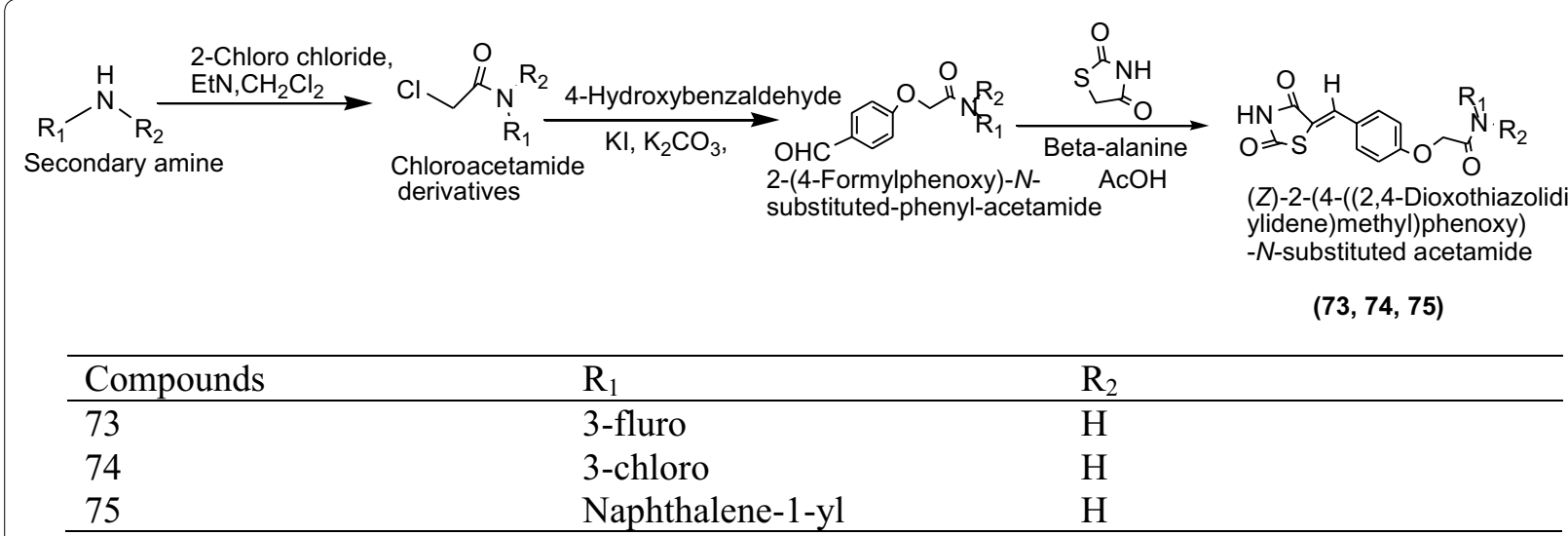

Scheme 23 Synthesis of (Z)-2-(4-((2,4-Dioxothiazolidin-5-ylidene)methyl)phenoxy)-N-substituted acetamide

Table 24 Anti-inflammatory activities of synthesized derivatives

\begin{tabular}{ll}
\hline Compounds & No inhibition (\%) \pm SD \\
\hline $\mathbf{7 3}$ & $41.5 \pm 3.1$ \\
$\mathbf{7 4}$ & $80.9 \pm 5.0$ \\
$\mathbf{7 5}$ & $70.9 \pm 13.6$ \\
Indomethacin & $63.2 \pm 4.0$ \\
\hline
\end{tabular}

from natural products have been isolated and developed as anticancer agents. These chemical compounds are formulated with a view to create effective action with minimum side effects against cancer [32].
Patil et al. [33] developed a novel class of 5-benzylidene-2,4-thiazolidinediones using Scheme 24. The synthesized derivatives were screened for the anticancer activity against K-562 (human leukemia), MCF-7 (human breast cancer), HepG-2 (human hepatoma), PC-3 (human prostate cancer), GURAV (human oral cancer) and KB (human nasopharyngeal cancer) cell lines by SRB protein assay. Among this series, 76, 77, 78 and 79 displayed the most potent anticancer activity compared with doxorubicin. The results of synthesized compounds presented in Table 25 (Patil et al. [33]).

Anh et al. [34] designed a chain of novel chromony thiazolidinediones derived from knoevenagel condensation reaction between 3-formyl-7-methoxy chromone with

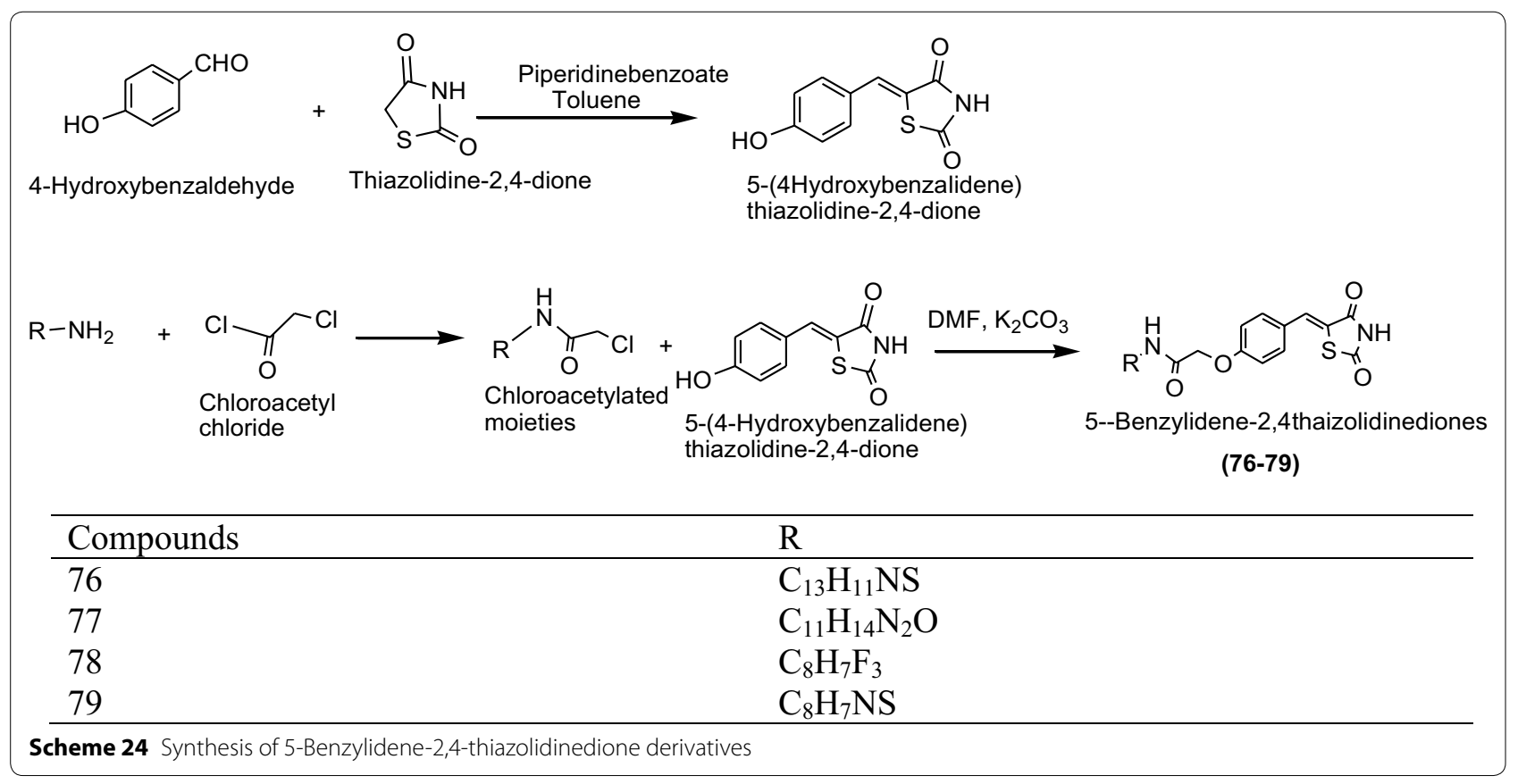


Table 25 Anti-tumor activities of synthesized derivatives in different cell lines

\begin{tabular}{|c|c|c|c|c|}
\hline Compounds & Diseases & Cancer cell line & $\log \mathrm{Gl}_{50}(\mu \mathrm{M})$ & $\log _{10} \mathrm{TGI}(\mu \mathrm{M})$ \\
\hline \multirow[t]{7}{*}{76} & Leukemia & $K-562$ & $>-0.4$ & $>-4.0$ \\
\hline & Breast cancer & MCF-7 & -4.53 & $>4.0$ \\
\hline & Hepatoma & HEPG-2 & $>-4.0$ & $>4.0$ \\
\hline & NSC lung cancer & HOP-62 & -6.72 & -4.54 \\
\hline & Prostate cancer & $P C-3$ & -4.53 & $>-4.0$ \\
\hline & Oral cancer & GURAV & $>-4.0$ & $>-4.0$ \\
\hline & Nasopharyngeal cancer & KB & $>-4.0$ & $>-4.0$ \\
\hline \multirow[t]{7}{*}{77} & Leukemia & K-562 & $>-4.0$ & $>-4.0$ \\
\hline & Breast cancer & MCF-7 & $>-4.0$ & $>-4.0$ \\
\hline & Hepatoma & HEPG-2 & $>-4.0$ & $>-4.0$ \\
\hline & NSC lung cancer & HOP-62 & -6.73 & $>-4.0$ \\
\hline & Prostate cancer & $P C-3$ & $>-4.0$ & $>-4.0$ \\
\hline & Oral cancer & GURAV & $>-4.0$ & $>-4.0$ \\
\hline & Nasopharyngeal cancer & KB & $>-4.0$ & $>-4.0$ \\
\hline \multirow[t]{7}{*}{78} & Leukemia & K-562 & -6.72 & $>-4.0$ \\
\hline & Breast cancer & MCF-7 & -6.71 & -4.52 \\
\hline & Hepatoma & HEPG-2 & $>-4.0$ & $>-4.0$ \\
\hline & NSC lung cancer & HOP-62 & $>-4.0$ & $>-4.0$ \\
\hline & Prostate cancer & $P C-3$ & -5.60 & $>-4.0$ \\
\hline & Oral cancer & GURAV & -6.73 & -4.52 \\
\hline & Nasopharyngeal cancer & KB & -5.65 & $>-4.0$ \\
\hline \multirow[t]{7}{*}{79} & Leukemia & K-52 & $>-4.0$ & $>-4.0$ \\
\hline & Breast cancer & MCF-7-5 & -4.60 & $>-4.0$ \\
\hline & Hepatoma & HEPG-2 & $>-4.0$ & $>-4.0$ \\
\hline & NSC lung cancer & HOP-62 & -6.77 & $>-4.0$ \\
\hline & Prostate cancer & $P C-3$ & -4.55 & -4.54 \\
\hline & Oral cancer & GURAV & $>-4.0$ & $>-4.0$ \\
\hline & Nasopharyngeal cancer & KB & $>-4.0$ & $>-4.0$ \\
\hline \multirow[t]{7}{*}{ Doxorubicin } & Leukemia & K-562 & -5.59 & $>-4.0$ \\
\hline & Breast cancer & MCF-7 & -6.88 & -5.68 \\
\hline & Hepatoma & HEPG-2 & $>-7.0$ & -6.87 \\
\hline & NSC lung cancer & HOP-62 & -6.91 & -4.45 \\
\hline & Prostate cancer & $P C-3$ & -6.96 & -5.68 \\
\hline & Oral cancer & GURAV & -6.97 & -6.80 \\
\hline & Nasopharyngeal cancer & KB & $>-7.0$ & -6.85 \\
\hline
\end{tabular}

different thiazolidinedione derivatives as presented in Scheme 25. These synthesized derivatives were screened for their cytotoxic activity against $\mathrm{Hep}-\mathrm{G}_{2}$ (heptocellular carcinoma), HC-60 (acute promyeloid carcinoma), KB (epidermoid carcinoma), LLC (lewis lung carcinoma), LNCaP (hormone dependent prostate carcinoma), MCF-7 (breast cancer), SW-480 (colon adenocarcinoma) cell lines using the MTT [3-(4,5-dimethylthiazol-2-yl)2,5-diphenyl- $2 H$-tetrazolium bromide] assay. In this series compounds 80, 81 and 82 showed highest cytotoxic activity against cancer cell lines. The results of potent compounds are presented in Table 26 (Anh et al. [34]).
Kumar et al. [35] synthesized a series of novel 3-(substituted aryl)-1-phenyl-1H-pyrazolyl-2,4-thiazolidinedione derivatives using Scheme 26. These synthesized derivatives were screened for their cytotoxic activity against lung and breast cancer cell lines using standard doxil. In this series $\mathbf{8 3}$ and $\mathbf{8 4}$ showed highest cytotoxic activity against cancer cell lines. The results of potent compounds are presented in Table 27 (Kumar et al. [35]).

\section{Thiazolidinedione derivatives as antioxidant agent}

Free radicals produced in several biochemical reactions, cellular metabolism are negotiator for several infections 


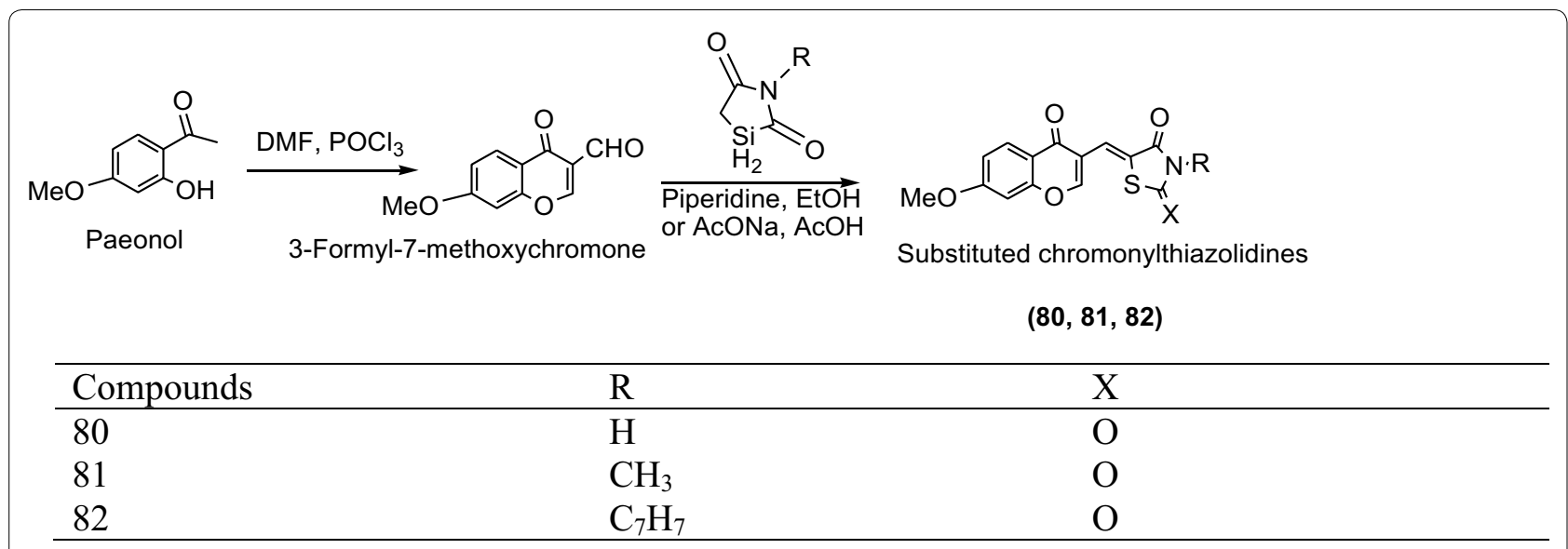

Scheme 25 Synthesis of 5-((7-Methoxy-4-oxo-4H-chromen-3-yl)methylene) substituted thiazolidine-2,4-dione

Table 26 Cytotoxicity of synthesized thiazolidinediones

\begin{tabular}{|c|c|c|c|c|c|c|c|c|}
\hline \multirow[t]{2}{*}{ Compounds } & \multicolumn{8}{|l|}{$I C_{50}(\mu \mathrm{g} / \mathrm{ml})$} \\
\hline & $\mathrm{HepG}_{2}$ & HC-60 & KB & LLC & LNCaP & LU-1 & MCF-7 & SW-480 \\
\hline 80 & $>100$ & $82.2 \pm 4.5$ & $44.1 \pm 3.6$ & $87.4 \pm 6.3$ & $77.4 \pm 5.8$ & $52.9 \pm 3.4$ & $66.0 \pm 2.7$ & $71.4 \pm 3.6$ \\
\hline 81 & $86.3 \pm 6.4$ & $75.3 \pm 3.9$ & $84.6 \pm 4.2$ & $>100$ & $81.6 \pm 6.3$ & $>100$ & $32.8 \pm 1.4$ & $90.1 \pm 4.8$ \\
\hline 82 & $78.4 \pm 5.8$ & $92.3 \pm 5.3$ & $74.1 \pm 5.1$ & $90.1 \pm 7.7$ & $84.2 \pm 4.1$ & $65.5 \pm 4.1$ & $52.7 \pm 3.6$ & $85.4 \pm 7.4$ \\
\hline Ellipticine & $1.45 \pm 0.08$ & $0.56 \pm 0.04$ & $0.43 \pm 0.05$ & $0.98 \pm 0.04$ & $0.86 \pm 0.06$ & $1.29 \pm 0.11$ & $0.49 \pm 0.04$ & $0.64 \pm 0.05$ \\
\hline
\end{tabular}

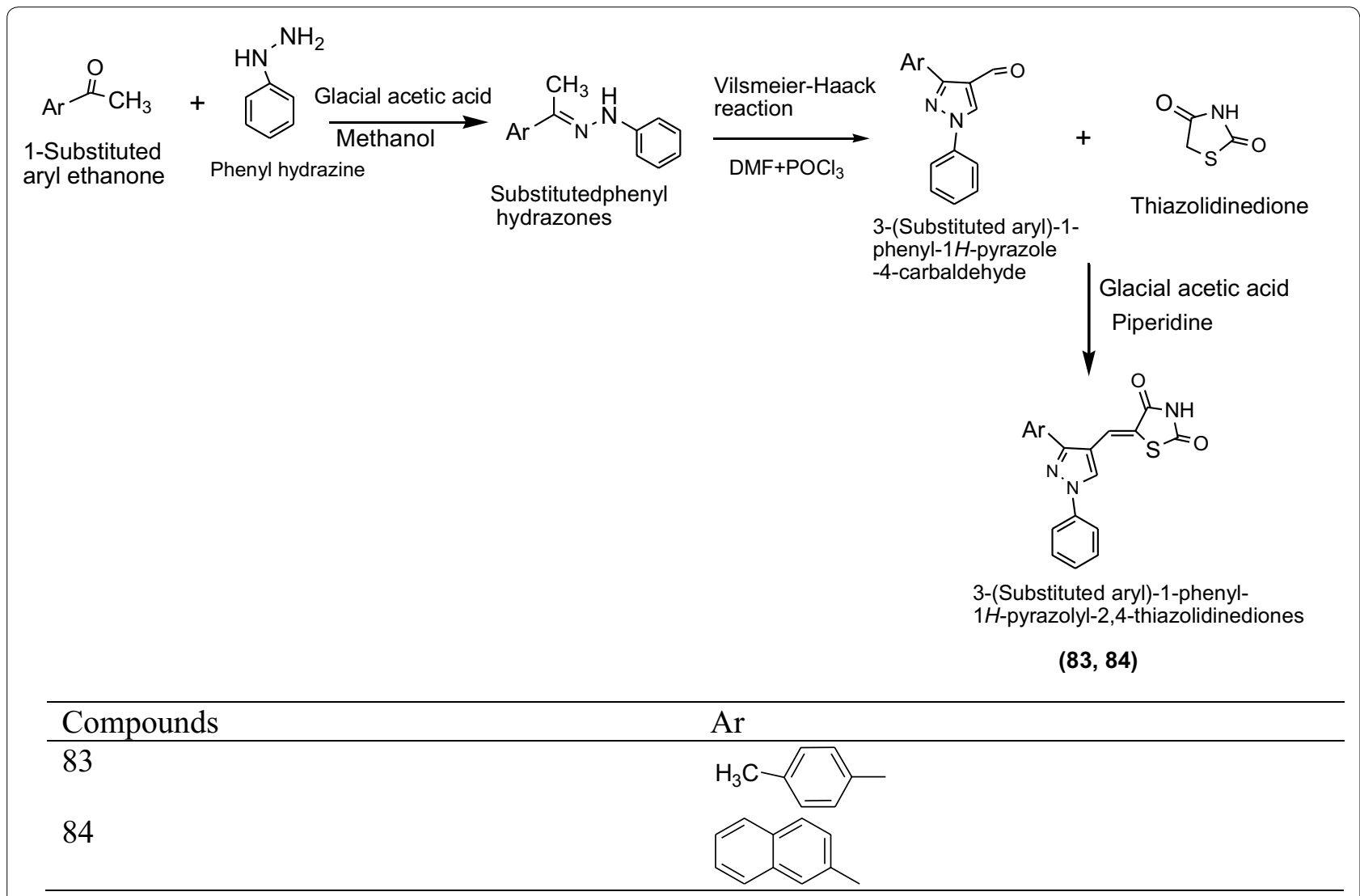

Scheme 26 Synthesis of 3-(Substituted aryl)-1-phenyl-1H-pyrazolyl-2, 4-thiazolidinediones 
Table $27 \mathrm{IC}_{50}$ value of synthesized derivatives against cancer cell lines

\begin{tabular}{llcc}
\hline Compounds & $\mathrm{IC}_{\mathbf{5 0}}(\boldsymbol{\mu M})$ & & \\
\cline { 2 - 4 } & A549 & MCF-7 & DU145 \\
\hline $\mathbf{8 3}$ & 05.12 & 09.16 & 43.17 \\
$\mathbf{8 4}$ & 06.83 & 4.44 & 59.29 \\
Doxil & 07.92 & 08.12 & 07.22 \\
\hline
\end{tabular}

and diseases like atherosclerosis, tumor as well as heart disease. Free radicals are not only formed by normal cellular processes but also produced by exposure of numerous chemical substances (polycyclic aromatic hydrocarbon, cadmium, lead, etc.), radiations, cigarette, smoke, and higher obese food. Usually free radical development is stopped by beneficial compounds known as antioxidant. Antioxidants deactivate free radicals before they attack the cell. Natural antioxidants are body detoxifiers and natural cleansers. They convert toxins of body to harmless waste products. They protect body from many diseases like cancer, heart attack and absorb bad cholesterol. Synthetic antioxidants such as BHT (butylated hydroxytoluene) and BHA (butylated hydroxyanisole), are effective as a antioxidants are also present and are used in several industries but there use has been limited because they can cause cancer as well as other side effects. So there use is decreased in food, cosmetic and pharmaceutical products. Thus, in present there is need for the oxidation inhibitor compounds [18, 36, 37].

Hossain et al. [37] synthesized a series of novel $O$-prenylated and $O$-geranylated derivatives of 5-benzylidene2,4-thiazolidinedione by knoevengeal condensation as showed in Scheme 27 and evaluated for their antioxidant activity. Among the synthesized derivatives, compounds $85,86,87,88$ and 89 were found to be most active antioxidant agent. The significant results of potent compounds are given in Table 28 (Hossain et al. [37]).
Lupascu et al. [4] designed a chain of novel thiazolidinediones containing xanthine moiety (Scheme 28) and evaluated for antioxidant potential using in vitro models such as DPPH radical scavenging assay and ABTS [2,2-azino-bis-(3-ethyl benzothiazoline-6-sulfonic acid] radical scavenging assay method. Among the synthesized derivatives 90, 91, 92 and 93 showed highest antioxidant activity. The results of potent derivatives are given in Table 29 (Lupascu et al. [4]).

\section{Thiazolidinedione derivatives as anti-tubercular agents} In present day, treatment of tuberculosis diseases (TB) is chief and challenging problem because of resistance to present regimen and also appearance of drug-resistance strains in tuberculosis like mycobacterium tuberculosis, is transmitted by air and can affected all organ of the body, especially the lungs [38]. The association of tuberculosis with HIV infection is so dramatic that in some cases, nearly two-third of the patients diagnosed with the tuberculosis is also HIV-1 seropositive [39]. The current drug therapy for TB is long and complex, involving multidrug combinations (usually isoniazid, rifampin, ethambutol, and pyrazinamide for the initial 2 months and rifampin and isoniazid for an additional 4 months) [40]. There is also an alarming increase in cases of TB caused by multidrug-resistant strains of M. tuberculosis. Thus,

Table 28 Inhibition of DPPH radical by synthesized compounds

\begin{tabular}{lllllc}
\hline Compounds & $\mathbf{R}_{\mathbf{1}}$ & $\mathbf{R}_{\mathbf{2}}$ & $\mathbf{R}_{\mathbf{3}}$ & $\mathbf{R}_{\mathbf{4}}$ & $\mathbf{I C}_{\mathbf{5 0}}(\boldsymbol{\mu} \mathbf{M})$ \\
\hline a-Tocopherol & $\mathrm{H}$ & Hydroxyl & $\mathrm{H}$ & $\mathrm{H}$ & 2.3 \\
$\mathbf{8 5}$ & Methoxy & Hydroxyl & $\mathrm{H}$ & $\mathrm{H}$ & 2.49 \\
$\mathbf{8 6}$ & Methoxy & Hydroxyl & Methoxy & $\mathrm{H}$ & 2.85 \\
$\mathbf{8 7}$ & Methoxy & PRO & $\mathrm{H}$ & $\mathrm{H}$ & 17.89 \\
$\mathbf{8 8}$ & Methoxy & PRO & Methoxy & $\mathrm{H}$ & 4.08 \\
$\mathbf{8 9}$ & $\mathrm{H}$ & $\mathrm{GRO}$ & $\mathrm{H}$ & $\mathrm{H}$ & 9.8
\end{tabular}

DPPH 1,1-diphenyl-2-picrylhydrazyl

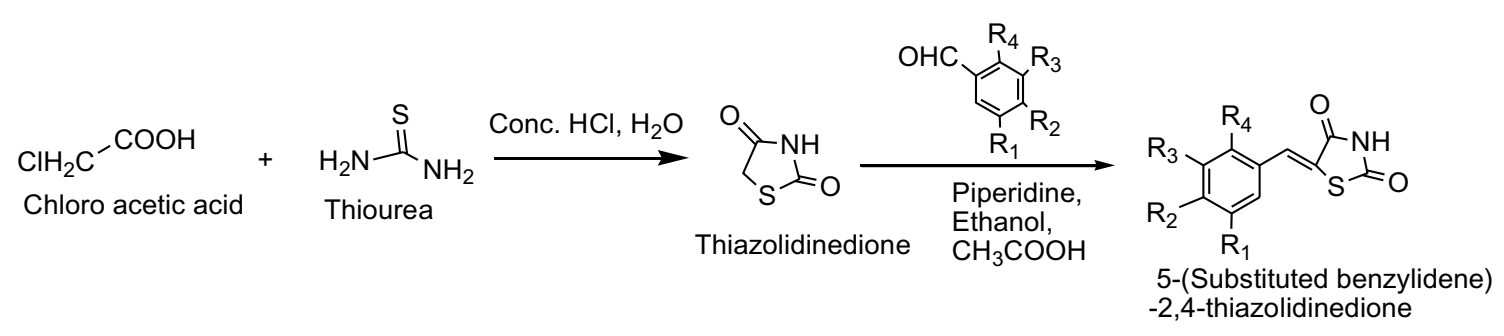

(85-89) 


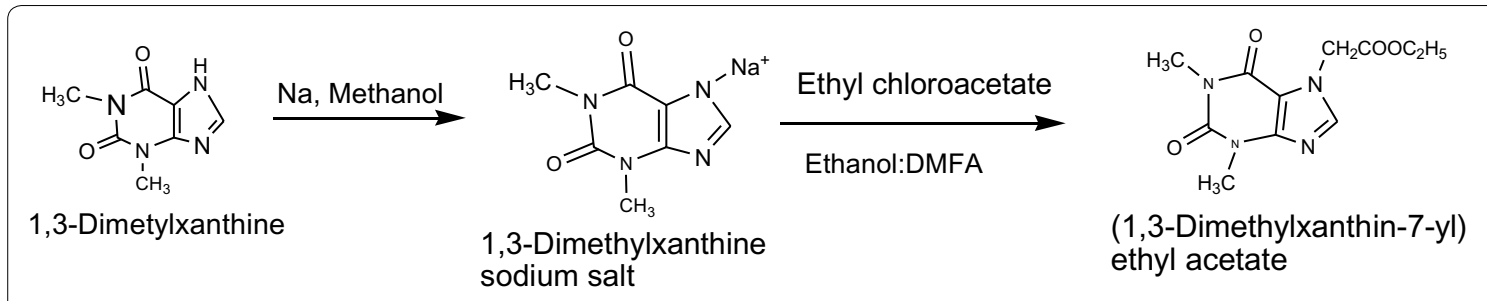<smiles></smiles>

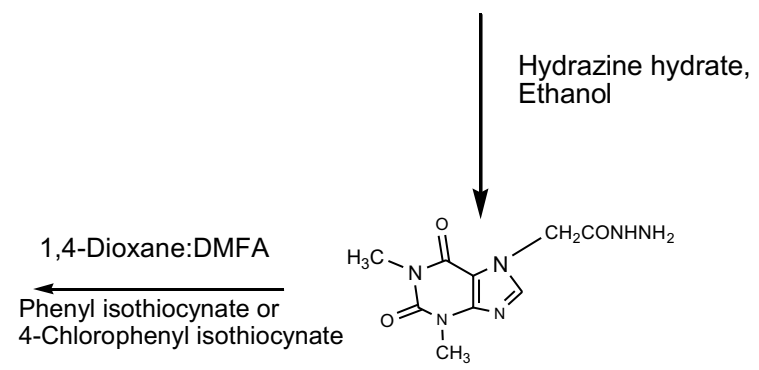

Thiosemicarbazide derivatives

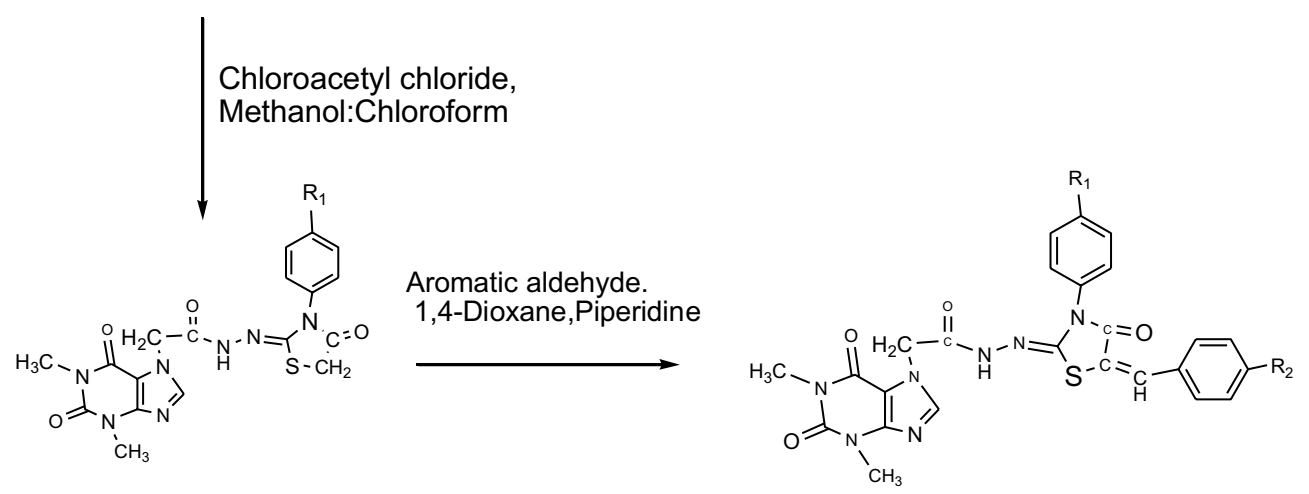

Thiazolidin-4-one derivatives

Benzylidenethiazolidin-4-one derivatives

\begin{tabular}{lll}
\hline Compounds & $\mathrm{R}_{1}$ & $\mathrm{R}_{2}$ \\
\hline 90 & $\mathrm{H}$ & 4-hydroxy \\
91 & $\mathrm{H}$ & $4-\mathrm{N}\left(\mathrm{CH}_{3}\right)_{2}$ \\
92 & $\mathrm{Cl}$ & $4-\mathrm{hydroxy}$ \\
93 & $\mathrm{Cl}$ & $4-\mathrm{N}\left(\mathrm{CH}_{3}\right)_{2}$ \\
\hline
\end{tabular}

Scheme 28 Synthesis of 2-\{2-[2-(1,3-Dimethylxanthin-7-yl)acetyl]hydrazono\}-3-(4-R1-phenyl-5-(R2-benzyliden)thiazolidin-4-ones

Table 29 Antioxidant activities of the synthesized derivatives

\begin{tabular}{lc}
\hline Compounds & $\mathrm{EC}_{\mathbf{5 0}} \mathbf{~ m g / m l}$ \\
\hline $\mathbf{9 0}$ & $0.025 \pm 0.0012$ \\
$\mathbf{9 1}$ & $0.022 \pm 0.0013$ \\
$\mathbf{9 2}$ & $0.033 \pm 0.0014$ \\
$\mathbf{9 3}$ & $0.026 \pm 0.0028$ \\
Ascorbic acid & $0.0067 \pm 0.0003$ \\
\hline
\end{tabular}

there is a need for new drugs targeting enzymes essential to mycobacterium survival $[41,42]$.

Chilamakuru et al. [42] synthesized a series of novel 3,5-disubstituted-2,4-thiazolidinediones as presented in Scheme 29 and appraised for anti-tubercular activities with pyrazinamide and streptomycin as the standard drug. Among all the synthesized derivatives, compounds 94, 95 [3-(2-amino-5-nitrophenyl)-5-(4methoxybenzylidene)-1,3-thiazolidine-2,4-dione], 


\begin{tabular}{|llll}
\hline Chloro acetic acid \\
Thiourea
\end{tabular}

Scheme 29 Synthesis of 3,5-Disubstituted-1,3-thiazolidine-2,4-dione

[3-tert-butyl-5-(4-methoxybenzylidene)-1,3-thiazolidine2,4-dione] and 97 showed the maximum antitubercular activity against Mycobacterium tuberculosis H37Rv strain. The results of synthesized compounds presented in Table 30 (Chliamakuru et al. [42]).

Pattan et al. [43] integrating a series of novel substituted thiazolidinediones via knoevenageal condensation reaction as presented in Scheme 30 and evaluated for their antitubercular activites by middle book 7H9 agar medium assay with streptomycin as the standard drug. Among all the synthesized derivatives, compounds 98 [(Z)-N-(3(4-((2,4-dioxothiazolidin-5-ylidene)methyl)phenoxy)2-oxopropyl)pyrazin-2-carboxamide] and [(Z)-5-(4-methoxybenzylidene)-3-(2-oxo-2-(pyrazin2-yl)ethyl)thiazolidine-2,4-dione] showed the maximum antitubercular activity against Mycobacterium tuberculosis $\mathrm{H} 37 \mathrm{Rv}$ strain. The results of synthesized compounds presented in Table 31 (Pattan et al. [43]).
Table 30 Anti-tubercular activity of synthesized derivatives

\begin{tabular}{ll}
\hline Compounds & MIC $\boldsymbol{\mu g} \mathbf{~} \mathbf{m l}$ \\
\hline $\mathbf{9 4}$ & 12.5 \\
$\mathbf{9 5}$ & 12.5 \\
$\mathbf{9 6}$ & 12.5 \\
$\mathbf{9 7}$ & 12.5 \\
Pyrazinamide & 3.125 \\
Streptomycin & 6.25 \\
\hline
\end{tabular}

\section{Conclusion}

Appraisal of literature reports reveals that thiazolidinediones and its derivatives represent an important class of compound in the medicinal field with various therapeutic potentials, i.e., antidiabetic, antimicrobial, 


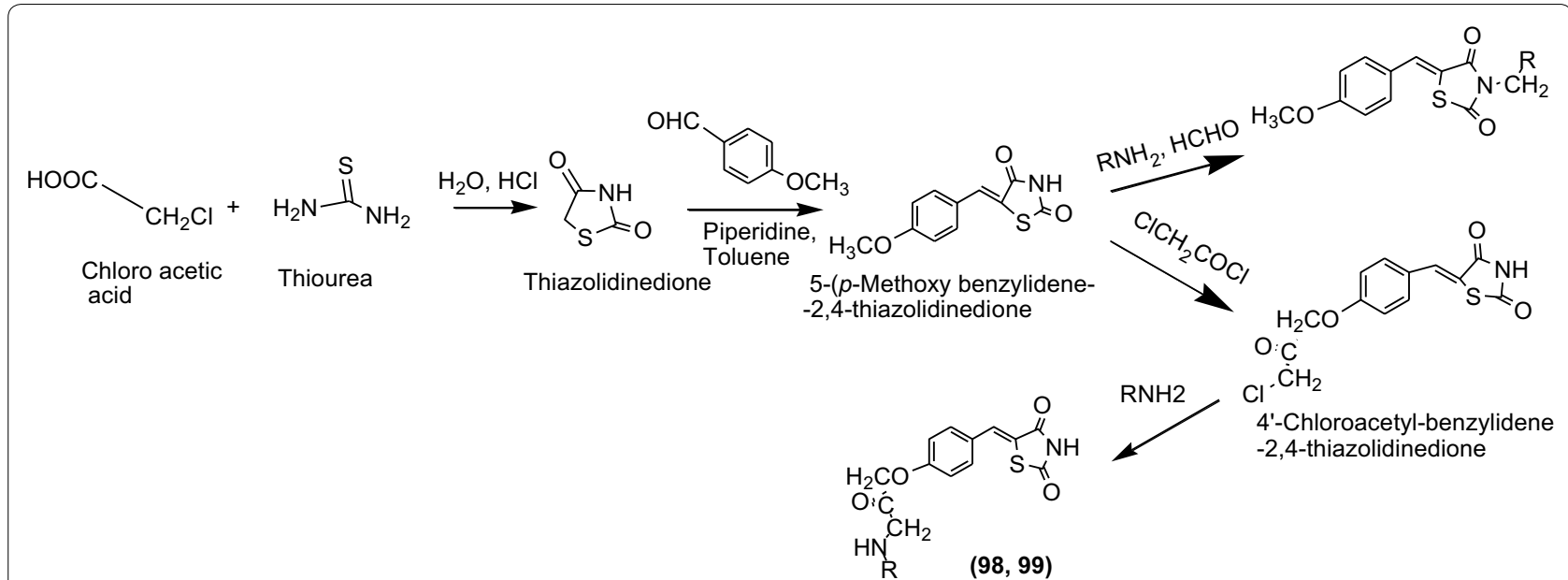

Compounds 98

Scheme 30 Synthesis of 4-Substitutedacetyl-benzylidene-2,4-thiazolidinediones

Table 31 Antitubercular activity of synthesized derivatives

\begin{tabular}{llll}
\hline Compounds & $\mathbf{2 5} \boldsymbol{\mu \mathbf { g } / \mathbf { m l }}$ & $\mathbf{5 0} \boldsymbol{\mu \mathbf { g } / \mathbf { m l }}$ & $\mathbf{1 0 0} \boldsymbol{\mu \mathbf { g } / \mathbf { m l }}$ \\
\hline $\mathbf{9 8}$ & Resistant & Resistant & Sensitive \\
$\mathbf{9 9}$ & Resistance & Resistance & Sensitive \\
Streptomycin & Sensitive & Sensitive & Sensitive \\
\hline
\end{tabular}

anti-inflammatory, anticancer, antioxidant and antitubercular, antiviral, anti-malarial, anti-HIV and anti-convulsant activities etc. which created immense interest among researchers to synthesized variety of thiazolidinediones. This review focuses especially on synthesized active compounds of thiazolidinediones having different pharmacological activities playing an important role in the medicinal field. These most active thiazolidinediones derivatives may be taken as leads to discover novel agents with therapeutic potential in the future.

\section{Authors' contributions}

PKV designed and finalized the scheme; SA performed review work and ST wrote the paper. All authors read and approved the final manuscript.

\section{Acknowledgements}

Thanks to Head, Department of Pharmaceutical Sciences, M. D. University, Rohtak for kind support for providing internet facilities etc.
Competing interests

The authors declare that they have no competing interests.

Ethics approval and consent to participate

Not applicable.

\section{Publisher's Note}

Springer Nature remains neutral with regard to jurisdictional claims in published maps and institutional affiliations.

Received: 13 October 2017 Accepted: 23 November 2017

Published online: 08 December 2017

\section{References}

1. Yang Y, Hu X, Zhang Q, Zou R (2016) Diabetes mellitus and risk of fall in older adult: a systematic review and meta-analysis. Age Ageing 45(6):761-767

2. Pattan SR, Kekare P, Patil A, Nikalje A, Kittur BS (2009) Studies on the synthesis of novel 2,4-thiazolidinedione derivatives with ant diabetic activity. Iran J Pharm Sci 5(4):225-232

3. Rekha S, Shantharam U, Chandy V (2011) Synthesis and evaluation of novel thiazolidinedione anti-inflammatory activity. Int Res J Pharm 2(9):81-84

4. Lupascu FG, Dragostin OM, Foia L, Lupascu D (2013) The synthesis and the biological evaluation of new thiazolidin-4-one derivatives containing a xanthine moiety. Lenuta Profire Mol 18:9684-9703

5. Moorthy P, Ekambaram SP, Perumal SS (2014) Synthesis, characterization and antimicrobial evaluation of imidazolyl thiazolidinedione derivatives. Arabian J Chem 8:1-7

6. Unlusoy MC, Dundar OB, Alanlar N, Ertan R (2006) Synthesis and antimicrobial activity of some new 3-substituted 
benzyl-5-(4-chloro-2-piperidin-1-ylthiazole-5-yl-methylene)-thiazolidine2,4-dione derivatives. Turk J Chem 30:355-360

7. Datar PA, Aher SB (2016) Desigh and synthesis of novel thiazoldine-2,4-dione as hypoglycemic agents. J Saudi Chem Soc 196-210

8. Swapna D, Sivagani B, Manasa K, Rajita G, Alagarsamy V (2016) Synthesis and evaluation of novel thiazolidinedione derivatives for antidiabetic activity. Int Res J Pharma 15-19

9. Badiger NP, Shashidhar N, Vaidya PN (2015) Synthesis of novel 5-\{[2-(4-fluorobenzyl)-6-arylimidazo[2,1-b] [1,3,4]thiadiazol-5-yl]methylene\}thiazolidine-2,4-diones as potent antidiabetic agents. Int J Sci Eng Appl 4(2):24-29

10. Patil SD, Nawale SL, Balasurbramaniyan V (2015) Evaluation of thiazolidinedione derivatives for acute toxicity and potential antidiabetic activity. Der Pharm Chem 7(5):216-223

11. Srikanth L, Raghunandan N, Srinivas P, Reddy GA (2010) Synthesis and evaluation of newer quinoline derivatives of thiazolidinedione for their ant diabetic activity. Int J Pharm Bio Sci 1:120-131

12. Nikaljea PGA, Choudharia S, Une H (2012) Design, synthesis and hypoglycemic activity of novel 2-(4-((2, 4-dioxothiazolidin-5-ylidene) methyl)2-methoxyphenoxy)- $\mathrm{N}$-substituted acetamide derivatives. Pelagia Res Library 2:1302-1314

13. Jiwane SK, Singh VK, Namdeo KP, Prajpap SK (2009) Synthesis of some novel 2,4-thiazolidinedione derivatives and their biological screening as ant diabetic agents. Asian J Chem 21:5068-5072

14. Grag A, Chawla P, Shubhini SA (2012) Substituted-arylidene-3-substituted-benzyl-thiazolidine-2,4-dione analogues as anti-hyperglycemic agents. Int J Drug Dev Res 4(3):141-146

15. Bhat BA, Ponnala S, SahuDP Tiwari P, Tripathi BK, Srivastava AK (2004) Synthesis and antihyperglycemic activity profiles of novel thiazolidinedione derivatives. Bioorg Med Chem 12:5857-5864

16. Jawale DV, Pratap UR, Rahuja N, Srivastava AK, Mane RA (2012) Synthesis and antihyperglycemic evaluation of new 2,4-thiazolidinediones having biodynamic aryl sulfonylurea moieties. Bioorg Med Chem 22:436-439

17. Vivekanand B, Mahendra Raj K, Mruthyunjayaswamy BHM (2015) Synthesis, characterization, antimicrobial, DNA-cleavage and antioxidant activities of 3-((5-chloro-2-phenyl-1 H-indol-3-ylimino)methyl)quinoline2(1H)-thione and its metal complexes. J Mol Struct 1079:214-224

18. Martin APM, Machado P, Piovesan LA, Flores AFC, De Campos MMA, Scheidt C, Bonacorso HG, Zanatta N (2008) Microwave-assisted synthesis and antimicrobial activity of 5-trihalomethyl-3-arylisoxazoles. MonatshChem 139:985-990

19. Nawale SL, Dhake AS (2012) Synthesis and evaluation of novel thiazolidinedione derivatives for antibacterial activity. Der Pharma Chemica 4(6):2270-2277

20. Nastasa CM, Duma M, Pirnau A, Vlase L, Tiperciuc B, Oniga O (2016) Development of new 5-(chromene-3-yl)methylene-2,4-thiazolidinediones as antimicrobial agents. Clujul Med 89(1):122-127

21. Alagawadi KR, Alegaon SG (2011) Synthesis, characterization and antimicrobial activity evaluation of new 2,4-thiazolidinediones bearing imidazo[2,1-b][1,3,4]thiadiazole moiety. Arabian J Chem 4:465-472

22. Khan FAK, Jadhav KS, Patil RH, Shinde DB, Arote RB, Sangshetti JN (2016) Biphenyl tetrazole-thiazolidinediones as novel bacterial peptide deformylase inhibitors: synthesis, biological evaluations and molecular docking study. Biomed Pharmacother 83:1146-1153

23. Liu XF, Zheng CJ, Sun LP, Liu XK, Piao HR (2011) Synthesis of new chalcone derivatives bearing 2,4-thiazolidinedione and benzoic acid moieties as potential anti-bacterial agents. Eur J Med Chem 46:3469-3473

24. Purohit SS, Alman A, Shewale J (2012) Synthesis and antimicrobial activity of a new series of 3,5-disustitutedthiazolidine-2,4-diones. Int J Pharm Pharm Sci 4(3):273-276

25. Sharma R, Vinay V (2012) Synthesis and antimicrobial activity of thiazolidinedione derivatives. Int J Sci Res Rev 1(1):57-66
26. Youssef AM, White MS, Villanueva EB, Ashmawy IM, Klegeris A (2010) Synthesis and evaluation of novel pyrazolyl-2,4-thiazolidinediones a anti-inflammatory and neuroprotective agents. Bioorg Med Chem 18:2019-2028

27. Dinarello CA (2010) Anti-inflammatory agents: present and future. Cell 140:935-950

28. Ma L, Xie C, Ma Y, Liu J, Xiang M, Ye X, Zheng H, Chen Z (2011) Synthesis and biological evaluation of novel 5 -benzylidenethiazolidine-derivatives 2,4-dione derivatives for the treatment of inflammatory diseases. J Med Chem 54:211-235

29. Dantu AS, Shankarguru P, Devi DR, Hari BNV (2012) Evaluation of in vitro anticancer activity of hydroalcohalicextract of Tabernaemontana divaricata. Asain J Pharm Clin Res 5(3):50-61

30. Arafa RK, Hegazy GH, Piazza GA, Abadi AH (2013) Synthesis and in vitro antiproliferative effect of novel quinoline-based potential anticancer agents. Eur J Med Chem 63:826-883

31. El-Damasy AK, Seo SH, Cho NC, Kang SB, Pae AN, Kim KS, Keum G (2013) Design, synthesis, in-vitro antiproliferative activity and kinase profile of new picolinamide based 2-amido and ureido quinoline derivatives. Eur J Med Chem 101:754-768. https://doi.org/10.1016/j.ejmech.2015.07.025

32. Merina N, Chandra KJ, Jibon K (2012) Medicinal plants with potential anticancer activities: a review. Int Res J Pharm 3(6):26-30

33. Patil V, Tilekar K, Munj SM, Mohan R, Ramaa CS (2010) Synthesis and primary cytotoxicity evaluation of new 5-benzylidene-2,4-thiazolidinedione derivatives. Eur J Med Chem 45:4539-4544

34. Anh HLT, CuC NT, Tai BH, Yen PH, Xuan N, Thao DT, Nam NH, Minh CV, Kiem PV, Kim YH (2015) Synthesis of chromonylthiazolidines and their cytotoxicity to human cancer cell lines. Molecules 20:1151-1160

35. Kumar KS, Reddy BM, Babu VH (2014) Synthesis of some novel 2,4-thiazolidinedione incorporated pyrazole derivatives as anticancer agents. Int J Pharm Sci 6(2):831-834

36. Feng L, Lv K, Liu M, Wang S, Zhao J, You X, Li S, Cao J, Guo H (2012) Eur J Med Chem 55:125-136

37. Hossain SU, Bhattachary S (2007) Synthesis of O-prenylared and O-geranylated derivatives of 5-benzylidene 2,4-thiazolidinediones and evaluation of their free radical scavenging activity as well s effect on some phase II antioxidant/detoxifying enzymes. Bioorg Med Chem Lett 17:1149-1154

38. Junior NS, Hyaric ML, Da Costa CF, Correa TA, Taveira AF, Araujo DP, Reis EFC, Lourenco MCS, Vicente FRC, De Almeida MV (2009) Preparation and antitubercular activity of lipophilic diamines and amino alcohols. Med Inst Oswaldo Cruz 104(5):703-705

39. Illango K, Arunkumar S (2011) Synthesis, antimicrobial and antitubercular activities of some novel trihydroxybenzamidoazetidin-2-one derivatives. Trop J Pharm Res 10(2):219-229

40. Thompson AM, Blaser A, Anderson RF, Shinde SS, Franzblau SG, Ma Z, Denny WA, Palmer BD (2008) Synthesis, reduction potential and antitubercular activity of ring $\mathrm{A} / \mathrm{B}$ analogues of the bioreductive drug (6S)2-nitro-6-\{[4-(trifluoromethoxy)benzyl]oxy\}-6,7-dihydro-5H-imidazo[2,1-b] [1,3]oxazine. J Med Chem 52(3):637-645

41. Bate $A B$, Kalin JH, Fooksman EM, Amorose EL, Price CM, Williams HM, Rodig MJ, Mitchell MO, Cho SH, Wang Y, Franzblau SG (2006) Synthesis and antitubercular activity of quaternized promazine and promethazine derivatives. Bioorg Med Chem 17(5):1346-1348

42. Chilamakuru N, Shankarananth V, Rajaskhar KK, Singirisetty T (2013) Synthesis, characterization and anti-tubercular activity of some new 3,5-disubstituted-2,4-thiazolidinediones. Asian J Pharm Clin Res 6(5):29-33

43. Pattan S, Kedar M, Pattan J, Dengale S, Sanap M, Gharate U, Shinde P, Kadam S (2012) Synthesis and evaluation of some novel 2,4-thiszolidinedione derivatives for antibacterial, antitubercular and antidiabetic activities. Indian J Chem 51B:1421-1425 